\title{
A Layer Correlation technique for pion energy calibration at the 2004 ATLAS Combined Beam Test
}

E. Abat,${ }^{k, 1}$ J.M. Abdallah, ${ }^{f}$ T.N. Addy, ${ }^{a g}$ P. Adragna,,$c c$ M. Aharrouche, ${ }^{b a}$

A. Ahmad, ${ }^{c m, 2}$ T.P.A. Akesson, ${ }^{a y}$ M. Aleksa, ${ }^{s}$ C. Alexa, ${ }^{n}$ K. Anderson, ${ }^{t}$

A. Andreazza, ${ }^{b e, b f}$ F. Anghinolfi, ${ }^{s}$ A. Antonaki, ${ }^{e}$ G. Arabidze, ${ }^{e}$ E. Arik, ${ }^{k}$ T. Atkinson, ${ }^{b d}$ J. Baines, ${ }^{c f}$ O.K. Baker, ${ }^{d d}$ D. Banfi, ${ }^{b e, b f}$ S. Baron, ${ }^{s}$ A.J. Barr, ${ }^{b s}$ R. Beccherle, ${ }^{a j}$

H.P. Beck, ${ }^{i}$ B. Belhorma, ${ }^{a w}$ P.J. Bell, ${ }^{b b, 3}$ D. Benchekroun, ${ }^{q}$ D.P. Benjamin, ${ }^{a c}$

K. Benslama, ${ }^{c g}$ E. Bergeaas Kuutmann, ${ }^{c p, 4}$ J. Bernabeu, ${ }^{c z}$ H. Bertelsen, ${ }^{v}$ S. Binet, ${ }^{b q}$

C. Biscarat, ${ }^{a d}$ V. Boldea, ${ }^{n}$ V.G. Bondarenko, ${ }^{b k}$ M. Boonekamp, ${ }^{c j}$ M. Bosman, ${ }^{f}$

C. Bourdarios, ${ }^{b q}$ Z. Broklova, ${ }^{c a}$ D. Burckhart Chromek, ${ }^{s}$ V. Bychkov, ${ }^{a n}$ J. Callahan, ${ }^{a i}$

D. Calvet, ${ }^{u}$ M. Canneri, ${ }^{b w}$ M. Capeáns Garrido, ${ }^{s}$ M. Caprini, ${ }^{n}$ L. Cardiel Sas, ${ }^{s}$

T. Carli, ${ }^{s}$ L. Carminati, ${ }^{b e}, b f$ J. Carvalho, ${ }^{p, b y}$ M. Cascella, ${ }^{b w}$ M.V. Castillo, ${ }^{c z}$

A. Catinaccio, ${ }^{s}$ D. Cauz, ${ }^{a k}$ D. Cavalli, ${ }^{b e}$ M. Cavalli Sforza, ${ }^{f}$ V. Cavasinni, ${ }^{b w}$

S.A. Cetin, ${ }^{k}$ H. Chen, ${ }^{j}$ R. Cherkaoui, ${ }^{c d}$ L. Chevalier,${ }^{c j}$ F. Chevallier, ${ }^{a w}$ S. Chouridou, ${ }^{c x}$

M. Ciobotaru, ${ }^{c v}$ M. Citterio, ${ }^{b e}$ A. Clark, ${ }^{a e}$ B. Cleland, ${ }^{b x}$ M. Cobal, ${ }^{a k}$ E. Cogneras, ${ }^{i}$

P. Conde Muino, ${ }^{b y}$ M. Consonni, ${ }^{b e, b f}$ S. Constantinescu, ${ }^{n}$ T. Cornelissen,,${ }^{s, 5}$

S. Correard, ${ }^{w}$ A. Corso Radu, ${ }^{s}$ G. Costa, ${ }^{b e}$ M.J. Costa, ${ }^{c z}$ D. Costanzo, ${ }^{c l}$ S. Cuneo, ${ }^{a j}$

P. Cwetanski, ${ }^{a i}$ D. Da Silva, ${ }^{c h}$ M. Dam, ${ }^{v}$ M. Dameri, ${ }^{a j}$ H.O. Danielsson, ${ }^{s}$

D. Dannheim, ${ }^{s}$ G. Darbo, ${ }^{a j}$ T. Davidek, ${ }^{c a}$ K. De, ${ }^{d}$ P.O. Defay, ${ }^{u}$ B. Dekhissi, ${ }^{a x}$ J. Del Peso, ${ }^{a z}$ T. Del Prete, ${ }^{b w}$ M. Delmastro, ${ }^{s}$ F. Derue, ${ }^{a v}$ L. Di Ciaccio, ${ }^{a r}$ B. Di Girolamo, ${ }^{s}$ S. Dita, ${ }^{n}$ F. Dittus, ${ }^{s}$ F. Djama, ${ }^{w}$ T. Djobava, ${ }^{c s}$ D. Dobos, ${ }^{a a, 6}$ M. Dobson, ${ }^{s}$

B.A. Dolgoshein, ${ }^{b k}$ A. Dotti, ${ }^{b w}$ G. Drake, ${ }^{b}$ Z. Drasal, ${ }^{c a}$ N. Dressnandt, ${ }^{b u}$ C. Driouchi, ${ }^{v}$ J. Drohan, ${ }^{c w}$ W.L. Ebenstein, ${ }^{a c}$ P. Eerola, ${ }^{a y, 7}$ I. Efthymiopoulos, ${ }^{s}$ K. Egorov, ${ }^{a i}$

T.F. Eifert, ${ }^{s}$ K. Einsweiler, ${ }^{h}$ M. El Kacimi, ${ }^{a s}$ M. Elsing, ${ }^{s}$ D. Emelyanov, ${ }^{c f, 8}$ C. Escobar, ${ }^{c z}$ A.I. Etienvre, ${ }^{c j}$ A. Fabich, ${ }^{s}$ K. Facius, ${ }^{,}$A.I. Fakhr-Edine, ${ }^{o}$ M. Fanti, ${ }^{b e}, b f$ A. Farbin, ${ }^{d}$ P. Farthouat, ${ }^{s}$ D. Fassouliotis,${ }^{e}$ L. Fayard, ${ }^{b q}$ R. Febbraro, ${ }^{u}$ O.L. Fedin, ${ }^{b v}$ A. Fenyuk, ${ }^{c b}$

\footnotetext{
${ }^{1}$ Deceased

${ }^{2}$ Now at SUNY, Stony Brook, United States of America

${ }^{3}$ Now at Université de Genève, Switzerland

${ }^{4}$ Now at DESY, Zeuthen, Germany

${ }^{5}$ Now at INFN Genova and Università di Genova, Italy

${ }^{6}$ Now at CERN

${ }^{7}$ Now at University of Helsinki, Finland

${ }^{8}$ Now at Joint Institute for Nuclear Research, Dubna, Russia
} 

D. Fergusson, ${ }^{h}$ P. Ferrari, ${ }^{s,}$ R. Ferrari, ${ }^{b t}$ B.C. Ferreira, ${ }^{c h}$ A. Ferrer,${ }^{c z}$ D. Ferrere,,${ }^{a e}$
G. Filippini, ${ }^{u}$ T. Flick, ${ }^{d c}$ D. Fournier ${ }^{b q}$ P. Francavilla, ${ }^{b w}$ D. Francis,${ }^{s}$ R. Froeschl, ${ }^{s}$
D. Froidevaux, ${ }^{s}$ E. Fullana, ${ }^{b}$ S. Gadomski, ${ }^{a e}$ G. Gagliardi, ${ }^{a j}$ P. Gagnon, ${ }^{a i}$ M. Gallas, ${ }^{s}$
B.J. Gallop, ${ }^{c f}$ S. Gameiro, ${ }^{s}$ K.K. Gan, ${ }^{b p}$ R. Garcia, ${ }^{a z}$ C. Garcia, ${ }^{c z}$ I.L. Gavrilenko, ${ }^{b j}$
C. Gemme, ${ }^{a j}$ P. Gerlach, ${ }^{d c}$ N. Ghodbane, ${ }^{u}$ V. Giakoumopoulou, ${ }^{e}$ V. Giangiobbe, ${ }^{b w}$
N. Giokaris, ${ }^{e}$ G. Glonti, ${ }^{a n}$ T. Goettfert, ${ }^{b m}$ T. Golling, ${ }^{h, 10}$ N. Gollub, ${ }^{s}$ A. Gomes, ${ }^{a t, a u, b y}$
M.D. Gomez, ${ }^{a e}$ S. Gonzalez-Sevilla, ${ }^{c z, 11}$ M.J. Goodrick, ${ }^{r}$ G. Gorfine, ${ }^{b o}$ B. Gorini, ${ }^{s}$
D. Goujdami, ${ }^{o}$ K-J. Grahn, ${ }^{a q,}{ }^{12}$ P. Grenier, ${ }^{u, 13}$ N. Grigalashvili, ${ }^{a n}$ Y. Grishkevich, ${ }^{b l}$
J. Grosse-Knetter, ${ }^{l, 14}$ M. Gruwe,${ }^{s}$ C. Guicheney, ${ }^{u}$ A. Gupta, ${ }^{t}$ C. Haeberli, ${ }^{i}$
R. Haertel, ${ }^{b m, 15}$ Z. Hajduk, ${ }^{y}$ H. Hakobyan, ${ }^{d e}$ M. Hance, ${ }^{b u}$ J.D. Hansen, ${ }^{y}$ P.H. Hansen, ${ }^{,}$
K. Hara, ${ }^{c u}$ A. Harvey Jr., ${ }^{a g}$ R.J. Hawkings, ${ }^{s}$ F.E.W. Heinemann, ${ }^{b s}$ A. Henriques
Correia, ${ }^{s}$ T. Henss, ${ }^{d c}$ L. Hervas, ${ }^{s}$ E. Higon, ${ }^{c z}$ J.C. Hill, ${ }^{r}$ J. Hoffman, ${ }^{z}$ J.Y. Hostachy, ${ }^{a w}$
I. Hruska, ${ }^{c a}$ F. Hubaut, ${ }^{w}$ F. Huegging, ${ }^{l}$ W. Hulsbergen, ${ }^{s, 16}$ M. Hurwitz, ${ }^{t}$
L. Iconomidou-Fayard, ${ }^{b q}$ E. Jansen, ${ }^{c e}$ I. Jen-La Plante, ${ }^{t}$ P.D.C. Johansson, ${ }^{c l}$
K. Jon-And, ${ }^{c p}$ M. Joos, ${ }^{s}$ S. Jorgensen, ${ }^{f}$ J. Joseph, ${ }^{h}$ A. Kaczmarska, $,{ }^{y}, 17$ M. Kado, ${ }^{b q}$
A. Karyukhin, ${ }^{c b}$ M. Kataoka, ${ }^{s, 18}$ F. Kayumov, ${ }^{b j}$ A. Kazarov, ${ }^{b v}$ P.T. Keener, ${ }^{b u}$
G.D. Kekelidze, ${ }^{a n}$ N. Kerschen, ${ }^{c l}$ S. Kersten, ${ }^{d c}$ A. Khomich, ${ }^{b c}$ G. Khoriauli, ${ }^{a n}$
E. Khramov, ${ }^{a n}$ A. Khristachev, ${ }^{b v}$ J. Khubua, ${ }^{a n}$ T.H. Kittelmann, ${ }^{v}, 19$ R. Klingenberg, ${ }^{a a}$
E.B. Klinkby, ${ }^{a c}$ P. Kodys, ${ }^{c a}$ T. Koffas, ${ }^{s}$ S. Kolos, ${ }^{c v}$ S.P. Konovalov, ${ }^{b j}$
N. Konstantinidis, ${ }^{c w}$ S. Kopikov, ${ }^{c b}$ I. Korolkov, ${ }^{f}$ V. Kostyukhin, ${ }^{a j, 20}$ S. Kovalenko, ${ }^{b v}$
T.Z. Kowalski, ${ }^{x}$ K. Krüger, ${ }^{s, 21}$ V. Kramarenko, ${ }^{b l}$ L.G. Kudin, ${ }^{b v}$ Y. Kulchitsky, ${ }^{b i}$
C. Lacasta, ${ }^{c z}$ R. Lafaye, ${ }^{a r}$ B. Laforge,${ }^{a v}$ W. Lampl, ${ }^{c}$ F. Lanni, ${ }^{j}$ S. Laplace, ${ }^{a r} \mathbf{T}$. Lari, ${ }^{b e}$ A-C. Le Bihan,,$^{s, 22}$ M. Lechowski, ${ }^{b q}$ F. Ledroit-Guillon, ${ }^{a w}$ G. Lehmann, ${ }^{s}$ R. Leitner, ${ }^{c a}$
D. Lelas ${ }^{b q}$ C.G. Lester, ${ }^{r}$ Z. Liang, ${ }^{z}$ P. Lichard, ${ }^{s}$ W. Liebig, ${ }^{b o}$ A. Lipniacka, ${ }^{g}$
M. Lokajicek, ${ }^{b z}$ L. Louchard, ${ }^{u}$ K.F. Lourerio, ${ }^{b p}$ A. Lucotte, ${ }^{a w}$ F. Luehring, ${ }^{a i}$
B. Lund-Jensen, ${ }^{a q}$ B. Lundberg, ${ }^{a y}$ H. Ma, ${ }^{j}$ R. Mackeprang, ${ }^{v}$ A. Maio, ${ }^{a t, a u, b y}$ V.P. Maleev, ${ }^{b v}$ F. Malek, ${ }^{a w}$ L. Mandelli, ${ }^{b e}$ J. Maneira, ${ }^{b y}$ M. Mangin-Brinet, ${ }^{a e, 23}$

\footnotetext{
${ }^{9}$ Now at Nikhef National Institute for Subatomic Physics, Amsterdam, Netherlands

${ }^{10}$ Now at Yale University, New Haven, USA

${ }^{11}$ Now at Université de Genève, Switzerland

${ }^{12}$ Corresponding author

${ }^{13}$ Now at SLAC, Stanford, USA

${ }^{14}$ Now at Georg-August-Universität, Goettingen, Germany

${ }^{15}$ Now at Versicherungskammer Bayern, Munich, Germany

${ }^{16}$ Now at Nikhef National Institute for Subatomic Physics, Amsterdam, Netherlands

${ }^{17}$ Now at Université Pierre et Marie Curie (Paris 6) and Université Denis Diderot (Paris-7), France

${ }^{18}$ Now at Laboratoire de Physique de Particules (LAPP), Annecy-le-Vieux, France

${ }^{19}$ Now at University of Pittsburgh, USA

${ }^{20}$ Now at Physikalisches Institut der Universität Bonn, Germany

${ }^{21}$ Now at Universität Heidelberg, Germany

${ }^{22}$ Now at IPHC, Université de Strasbourg, CNRS/IN2P3, Strasbourg, France

${ }^{23}$ Now at Laboratoire de Physique Subatomique et de Cosmologie CNRS/IN2P3, Grenoble, France
} 
A. Manousakis, ${ }^{e}$ L. Mapelli,,${ }^{s}$ C. Marques, ${ }^{b y}$ S.Marti i Garcia, ${ }^{c z}$ F. Martin, ${ }^{b u}$

M. Mathes, ${ }^{l}$ M. Mazzanti, ${ }^{b e}$ K.W. McFarlane, ${ }^{a g}$ R. McPherson, ${ }^{d a}$ G. Mchedlidze, ${ }^{c s}$

S. Mehlhase, ${ }^{a h}$ C. Meirosu, ${ }^{s}$ Z. Meng, ${ }^{c k}$ C. Meroni, ${ }^{b e}$ V. Mialkovski, ${ }^{a n}$ B. Mikulec,${ }^{a e, 24}$

D. Milstead, ${ }^{c p}$ I. Minashvili, ${ }^{a n}$ B. Mindur, ${ }^{x}$ V.A. Mitsou, ${ }^{c z}$ S. Moed, ${ }^{a e, 25}$ E. Monnier, ${ }^{w}$

G. Moorhead, ${ }^{b d}$ P. Morettini, ${ }^{a j}$ S.V. Morozov, ${ }^{b k}$ M. Mosidze, ${ }^{c s}$ S.V. Mouraviev, ${ }^{b j}$

E.W.J. Moyse, ${ }^{s}$ A. Munar, ${ }^{b u}$ A. Myagkov, ${ }^{c b}$ A.V. Nadtochi, ${ }^{b v}$ K. Nakamura, ${ }^{c u, 26}$

P. Nechaeva, ${ }^{a j, 27}$ A. Negri, ${ }^{b t}$ S. Nemecek, ${ }^{b z}$ M. Nessi, ${ }^{s}$ S.Y. Nesterov, ${ }^{b v}$

F.M. Newcomer, ${ }^{b u}$ I. Nikitine, ${ }^{c b}$ K. Nikolaev, ${ }^{a n}$ I. Nikolic-Audit, ${ }^{a v}$ H. Ogren, ${ }^{a i}$ S.H. Oh, ${ }^{a c}$

S.B. Oleshko, ${ }^{b v}$ J. Olszowska, ${ }^{y}$ A. Onofre, $,{ }^{b g}, b y$ C. Padilla Aranda, ${ }^{s}$ S. Paganis, ${ }^{c l}$

D. Pallin, ${ }^{u}$ D. Pantea, ${ }^{n}$ V. Paolone, ${ }^{b x}$ F. Parodi, ${ }^{a j}$ J. Parsons, ${ }^{b n}$ S. Parzhitskiy, ${ }^{a n}$

E. Pasqualucci, ${ }^{c i}$ S.M. Passmored, ${ }^{s}$ J. Pater, ${ }^{b b}$ S. Patrichev, ${ }^{b v}$ M. Peez, ${ }^{a z}$ V. Perez Reale, ${ }^{b n}$ L. Perini, ${ }^{b e, b f}$ V.D. Peshekhonov, ${ }^{a n}$ J. Petersen, ${ }^{s}$ T.C. Petersen, ${ }^{v}$ R. Petti, ${ }^{j, 28}$ P.W. Phillips, ${ }^{c f}$ J. Pina, ${ }^{a t, a u, b y}$ B. Pinto, ${ }^{b y}$ F. Podlyski, ${ }^{u}$ L. Poggioli, ${ }^{b q}$ A. Poppleton, ${ }^{s}$ J. Poveda, ${ }^{d b}$ P. Pralavorio, ${ }^{w}$ L. Pribyl, ${ }^{s}$ M.J. Price,${ }^{s}$ D. Prieur, ${ }^{c f}$ C. Puigdengoles, ${ }^{f}$ P. Puzo, ${ }^{b q}$ O. Røhne, ${ }^{b r}$ F. Ragusa, ${ }^{b e, b f}$ S. Rajagopalan, ${ }^{j}$ K. Reeves, ${ }^{d c, 29}$ I. Reisinger, ${ }^{a a}$ C. Rembser, ${ }^{s}$ P.A.Bruckman.de. Renstrom, ${ }^{b s}$ P. Reznicek, ${ }^{c a}$ M. Ridel, ${ }^{a v}$ P. Risso, ${ }^{a j}$ I. Riu, ${ }^{a e, 30}$ D. Robinson, ${ }^{r}$ C. Roda, ${ }^{b w}$ S. Roe, ${ }^{s}$ O. Rohne, ${ }^{b r}$ A. Romaniouk, ${ }^{b k}$ D. Rousseau, ${ }^{b q}$ A. Rozanov, ${ }^{w}$ A. Ruiz, ${ }^{c z}$ N. Rusakovich, ${ }^{a n}$ D. Rust, ${ }^{a i}$ Y.F. Ryabov, ${ }^{b v}$ V. Ryjov, ${ }^{s}$ O. Salto, ${ }^{f}$ B. Salvachua, ${ }^{b}$ A. Salzburger, ${ }^{a l, 31}$ H. Sandaker, ${ }^{g}$ C. Santamarina Rios, ${ }^{s}$ L. Santi, ${ }^{a k}$ C. Santoni, ${ }^{u}$ J.G. Saraiva, ${ }^{a t, a u, b y}$ F. Sarri, ${ }^{b w}$ G. Sauvage,${ }^{a r}$ L.P. Says, ${ }^{,}$ M. Schaefer, ${ }^{a w}$ V.A. Schegelsky, ${ }^{b v}$ C. Schiavi, ${ }^{a j}$ J. Schieck, ${ }^{b m}$ G. Schlager, ${ }^{s}$ J. Schlereth, ${ }^{b}$ C. Schmitt, ${ }^{b a}$ J. Schultes, ${ }^{d c}$ P. Schwemling, ${ }^{a v}$ J. Schwindling, ${ }^{c j}$ J.M. Seixas, ${ }^{c h}$ D.M. Seliverstov, ${ }^{b v}$ L. Serin, ${ }^{b q}$ A. Sfyrla, ${ }^{a e, 32}$ N. Shalanda, ${ }^{b h}$ C. Shaw, ${ }^{a f}$ T. Shin, ${ }^{a g}$ A. Shmeleva, ${ }^{b j}$ J. Silva, ${ }^{b y}$ S. Simion, ${ }^{b q}$ M. Simonyan, ${ }^{a r}$ J.E. Sloper, ${ }^{s}$

S.Yu. Smirnov, ${ }^{b k}$ L. Smirnova, ${ }^{b l}$ C. Solans, ${ }^{c z}$ A. Solodkov, ${ }^{c b}$ O. Solovianov, ${ }^{c b}$ I. Soloviev, ${ }^{b v}$ V.V. Sosnovtsev, ${ }^{b k}$ F. Spanò, ${ }^{b n}$ P. Speckmayer, ${ }^{s}$ S. Stancu, ${ }^{c v}$ R. Stanek, ${ }^{b}$ E. Starchenko, ${ }^{c b}$ A. Straessner, ${ }^{a b}$ S.I. Suchkov, ${ }^{b k}$ M. Suk, ${ }^{c a}$ R. Szczygiel, ${ }^{x}$

F. Tarrade, ${ }^{j}$ F. Tartarelli, ${ }^{b e}$ P. Tas, ${ }^{c a}$ Y. Tayalati, ${ }^{u}$ F. Tegenfeldt, ${ }^{a m}$ R. Teuscher,${ }^{c t}$

M. Thioye ${ }^{c q}$ V.O. Tikhomirov, ${ }^{b j}$ C.J.W.P. Timmermans, ${ }^{c e}$ S. Tisserant, ${ }^{w}$ B. Toczek, ${ }^{x}$

L. Tremblet, ${ }^{s}$ C. Troncon, ${ }^{b e}$ P. Tsiareshka, ${ }^{b i}$ M. Tyndel, ${ }^{c f}$ M.Karagoez. Unel, ${ }^{b s}$

G. Unal, ${ }^{s}$ G. Unel, ${ }^{a i}$ G. Usai, ${ }^{t}$ R. Van Berg, ${ }^{b u}$ A. Valero, ${ }^{c z}$ S. Valkar, ${ }^{c a}$ J.A. Valls, ${ }^{c z}$ W. Vandelli, ${ }^{s}$ F. Vannucci, ${ }^{a v}$ A. Vartapetian, ${ }^{d}$ V.I. Vassilakopoulos, ${ }^{a g}$ L. Vasilyeva, ${ }^{b j}$

\footnotetext{
${ }^{24}$ Now at CERN

${ }^{25}$ Now at Harvard University, Cambridge, USA

${ }^{26}$ Now at ICEPP, Tokyo, Japan

${ }^{27}$ Now at P.N. Lebedev Institute of Physics, Moscow, Russia

${ }^{28}$ Now at University of South Carolina, Columbia, USA

${ }^{29}$ Now at UT Dallas

${ }^{30}$ Now at IFAE, Barcelona, Spain

${ }^{31}$ Now at CERN

${ }^{32}$ Now at CERN
} 
F. Vazeille, ${ }^{u}$ F. Vernocchi, ${ }^{a j}$ Y. Vetter-Cole, ${ }^{z}$ I. Vichou, ${ }^{c y}$ V. Vinogradov, ${ }^{a n}$ J. Virzi, ${ }^{,}$

I. Vivarelli, ${ }^{b w}$ J.B.de. Vivie, ${ }^{w, 33}$ M. Volpi, ${ }^{f}$ T. Vu Anh, ${ }^{a e, 34}$ C. Wang, ${ }^{a c}$ M. Warren, ${ }^{c w}$

J. Weber, ${ }^{a a}$ M. Weber, ${ }^{c f}$ A.R. Weidberg, ${ }^{b s}$ J. Weingarten, ${ }^{l, 35}$ P.S. Wells, ${ }^{s}$ P. Werner, ${ }^{s}$

S. Wheeler, ${ }^{a}$ M. Wiessmann, ${ }^{b m}$ H. Wilkens, ${ }^{s}$ H.H. Williams, ${ }^{b u}$ I. Wingerter-Seez, ${ }^{a r}$

Y. Yasu, ${ }^{a p}$ A. Zaitsev, ${ }^{c b}$ A. Zenin, ${ }^{c b}$ T. Zenis, ${ }^{m}$ Z. Zenonos, ${ }^{b w}$ H. Zhang, ${ }^{w}$ A. Zhelezko ${ }^{b k}$ and N. Zhou ${ }^{b n}$

${ }^{a}$ University of Alberta, Department of Physics, Centre for Particle Physics, Edmonton, AB T6G 2G7, Canada

${ }^{b}$ Argonne National Laboratory, High Energy Physics Division, 9700 S. Cass Avenue, Argonne IL 60439, United States of America

${ }^{c}$ University of Arizona, Department of Physics, Tucson, AZ 85721, United States of America

${ }^{d}$ University of Texas at Arlington, Department of Physics, Box 19059, Arlington, TX 76019, United States of America

${ }^{e}$ University of Athens, Nuclear \& Particle Physics Department of Physics, Panepistimiopouli Zografou, GR 15771 Athens, Greece

${ }^{f}$ Institut de Fisica d'Altes Energies, IFAE, Universitat Autònoma de Barcelona, Edifici Cn, ES - 08193 Bellaterra (Barcelona) Spain

${ }^{g}$ University of Bergen, Department for Physics and Technology, Allegaten 55, NO - 5007 Bergen, Norway

${ }^{h}$ Lawrence Berkeley National Laboratory and University of California, Physics Division, MS50B-6227, 1 Cyclotron Road, Berkeley, CA 94720, United States of America

${ }^{i}$ University of Bern, Laboratory for High Energy Physics, Sidlerstrasse 5, CH - 3012 Bern, Switzerland

${ }^{j}$ Brookhaven National Laboratory, Physics Department, Bldg. 510A, Upton, NY 11973, United States of America

${ }^{k}$ Bogazici University, Faculty of Sciences, Department of Physics, TR - 80815 Bebek-Istanbul, Turkey

${ }^{l}$ Physikalisches Institut der Universität Bonn, Nussallee 12, D - 53115 Bonn, Germany

${ }^{m}$ Comenius University, Faculty of Mathematics Physics \& Informatics, Mlynska dolina F2, SK - 84248 Bratislava, Slovak Republic

${ }^{n}$ National Institute of Physics and Nuclear Engineering (Bucharest-IFIN-HH), P.O. Box MG-6, R-077125 Bucharest, Romania

${ }^{o}$ Université Cadi Ayyad, Marrakech, Morocco

${ }^{p}$ Department of Physics, University of Coimbra, P-3004-516 Coimbra, Portugal

${ }^{q}$ Université Hassan II, Faculté des Sciences Ain Chock, B.P. 5366, MA - Casablanca, Morocco

${ }^{r}$ Cavendish Laboratory, University of Cambridge, J J Thomson Avenue, Cambridge CB3 OHE, United Kingdom

${ }^{s}$ European Laboratory for Particle Physics (CERN), CH-1211 Geneva 23, Switzerland

${ }^{t}$ University of Chicago, Enrico Fermi Institute, 5640 S. Ellis Avenue, Chicago, IL 60637, United States of America

${ }^{u}$ Laboratoire de Physique Corpusculaire (LPC), IN2P3-CNRS, Université Blaise-Pascal Clermont-Ferrand, FR - 63177 Aubiere, France

${ }^{v}$ Niels Bohr Institute, University of Copenhagen, Blegdamsvej 17, DK - 2100 Kobenhavn 0, Denmark

\footnotetext{
${ }^{33}$ Now at LAL-Orsay, France

${ }^{34}$ Now at Universität Mainz, Mainz, Germany

${ }^{35}$ Now at Georg-August-Universität, Goettingen, Germany
} 
${ }^{w}$ Université Méditerranée, Centre de Physique des Particules de Marseille, CNRS/IN2P3, F-13288 Marseille, France

${ }^{x}$ Faculty of Physics and Applied Computer Science of the AGH-University of Science and Technology, (FPACS, AGH-UST), al. Mickiewicza 30, PL-30059 Cracow, Poland

${ }^{y}$ The Henryk Niewodniczanski Institute of Nuclear Physics, Polish Academy of Sciences, ul. Radzikowskiego 152, PL - 31342 Krakow Poland

${ }^{z}$ Southern Methodist University, Physics Department, 106 Fondren Science Building, Dallas, TX $75275-$ 0175, United States of America

${ }^{a}$ Universität Dortmund, Experimentelle Physik IV, DE - 44221 Dortmund, Germany

${ }^{a b}$ Technical University Dresden, Institut für Kern- und Teilchenphysik, Zellescher Weg 19, D-01069 Dresden, Germany

${ }^{a c}$ Duke University, Department of Physics Durham, NC 27708, United States of America

${ }^{\text {ad }}$ Centre de Calcul CNRS/IN2P3, Lyon, France

${ }^{a}$ Université de Genève, Section de Physique, 24 rue Ernest Ansermet, CH - 1211 Genève 4, Switzerland

af University of Glasgow, Department of Physics and Astronomy, UK - Glasgow G12 8QQ, United Kingdom

${ }^{a g}$ Hampton University, Department of Physics, Hampton, VA 23668, United States of America

${ }^{a h}$ Institute of Physics, Humboldt University, Berlin, Newtonstrasse 15, D-12489 Berlin, Germany

ai Indiana University, Department of Physics, Swain Hall West 117, Bloomington, IN 47405-7105, United States of America

${ }^{a j}$ INFN Genova and Università di Genova, Dipartimento di Fisica, via Dodecaneso 33, IT - 16146 Genova, Italy

${ }^{a k}$ INFN Gruppo Collegato di Udine and Università di Udine, Dipartimento di Fisica, via delle Scienze 208, IT - 33100 Udine; INFN Gruppo Collegato di Udine and ICTP, Strada Costiera 11, IT - 34014 Trieste, Italy

${ }^{a l}$ Institut für Astro- und Teilchenphysik, Technikerstrasse 25, A - 6020 Innsbruck, Austria

${ }^{a m}$ Iowa State University, Department of Physics and Astronomy, Ames High Energy Physics Group, Ames, IA 50011-3160, United States of America

an Joint Institute for Nuclear Research, JINR Dubna, RU - 141980 Moscow Region, Russia

${ }^{a}$ Institut fuer Prozessdatenverarbeitung und Elektronik, Karlsruher Institut fuer Technologie, Campus Nord, Hermann-v.Helmholtz-Platz 1, D-76344 Eggenstein-Leopoldshafen

${ }^{a p}$ KEK, High Energy Accelerator Research Organization, 1-1 Oho Tsukuba-shi, Ibaraki-ken 305-0801, Japan

${ }^{a q}$ Royal Institute of Technology (KTH), Physics Department, SE - 10691 Stockholm, Sweden

${ }^{a r}$ Laboratoire de Physique de Particules (LAPP), Université de Savoie, CNRS/IN2P3, Annecy-le-Vieux Cedex, France

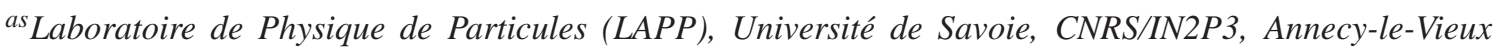
Cedex, France and Université Cadi Ayyad, Marrakech, Morocco

${ }^{a t}$ Departamento de Fisica, Faculdade de Ciências, Universidade de Lisboa, P-1749-016 Lisboa, Portugal

${ }^{a u}$ Centro de Física Nuclear da Universidade de Lisboa, P-1649-003 Lisboa, Portugal

av Université Pierre et Marie Curie (Paris 6) and Université Denis Diderot (Paris-7), Laboratoire de Physique Nucléaire et de Hautes Energies, CNRS/IN2P3, Tour 334 place Jussieu, FR - 75252 Paris Cedex 05, France

${ }^{a w}$ Laboratoire de Physique Subatomique et de Cosmologie CNRS/IN2P3, Université Joseph Fourier INPG, 53 avenue des Martyrs, FR - 38026 Grenoble Cedex, France

${ }^{a x}$ Laboratoire de Physique Théorique et de Physique des Particules, Université Mohammed Premier, Oujda, Morocco 
${ }^{a y}$ Lunds universitet, Naturvetenskapliga fakulteten, Fysiska institutionen, Box 118, SE - 221 00, Lund, Sweden

${ }^{a z}$ Universidad Autonoma de Madrid, Facultad de Ciencias, Departamento de Fisica Teorica, ES - 28049 Madrid, Spain

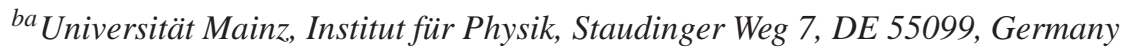

${ }^{b b}$ School of Physics and Astronomy, University of Manchester, UK - Manchester M13 9PL, United Kingdom

${ }^{b c}$ Universität Mannheim, Lehrstuhl für Informatik V, B6, 23-29, DE - 68131 Mannheim, Germany

${ }^{b d}$ School of Physics, University of Melbourne, AU - Parkvill, Victoria 3010, Australia

${ }^{b e}$ INFN Sezione di Milano, via Celoria 16, IT - 20133 Milano, Italy

bf Università di Milano, Dipartimento di Fisica, via Celoria 16, IT - 20133 Milano, Italy

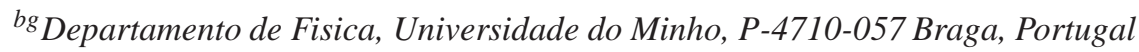

${ }^{b h}$ B.I. Stepanov Institute of Physics, National Academy of Sciences of Belarus, Independence Avenue 68, Minsk 220072, Republic of Belarus

${ }^{b i}$ B.I. Stepanov Institute of Physics, National Academy of Sciences of Belarus, Independence Avenue 68, Minsk 220072, Republic of Belarus and Joint Institute for Nuclear Research, JINR Dubna, RU - 141980 Moscow Region, Russia

${ }^{b j}$ P.N. Lebedev Institute of Physics, Academy of Sciences, Leninsky pr. 53, RU - 117 924, Moscow, Russia

${ }^{b k}$ Moscow Engineering \& Physics Institute (MEPhI), Kashirskoe Shosse 31, RU - 115409 Moscow, Russia

${ }^{b l}$ Lomonosov Moscow State University, Skobeltsyn Institute of Nuclear Physics, RU - 119991 GSP-1 Moscow Lenskiegory 1-2, Russia

${ }^{b m}$ Max-Planck-Institut für Physik, (Werner-Heisenberg-Institut), Föhringer Ring 6, 80805 München, Germany

${ }^{b n}$ Columbia University, Nevis Laboratory, 136 So. Broadway, Irvington, NY 10533, United States of America

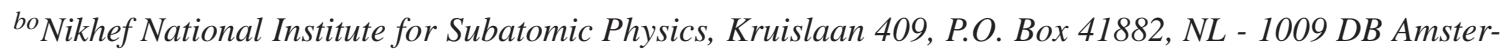
dam, Netherlands

${ }^{b p}$ Ohio State University, 191 West WoodruAve, Columbus, OH 43210-1117, United States of America

${ }^{b q}$ LAL, Université Paris-Sud, IN2P3/CNRS, Orsay, France

${ }^{b r}$ University of Oslo, Department of Physics, P.O. Box 1048, Blindern T, NO - 0316 Oslo, Norway

${ }^{b s}$ Department of Physics, Oxford University, Denys Wilkinson Building, Keble Road, Oxford OXI 3RH, United Kingdom

${ }^{b t}$ Università di Pavia, Dipartimento di Fisica Nucleare e Teorica and INFN Pavia, Via Bassi 6 IT-27100 Pavia, Italy

${ }^{b u}$ University of Pennsylvania, Department of Physics, High Energy Physics, 209 S. 33rd Street Philadelphia, PA 19104, United States of America

${ }^{b v}$ Petersburg Nuclear Physics Institute, RU - 188300 Gatchina, Russia

${ }^{b w}$ Università di Pisa, Dipartimento di Fisica E. Fermi and INFN Pisa, Largo B.Pontecorvo 3, IT - 56127 Pisa, Italy

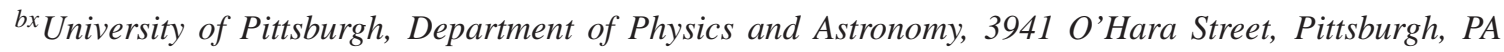
15260, United States of America

${ }^{b y}$ Laboratorio de Instrumentacao e Fisica Experimental de Particulas - LIP, and SIM/Univ. de Lisboa, Avenida Elias Garcia 14-1, PT - 1000-149, Lisboa, Portugal

${ }^{b z}$ Academy of Sciences of the Czech Republic, Institute of Physics and Institute for Computer Science, Na Slovance 2, CZ - 18221 Praha 8, Czech Republic

${ }^{c a}$ Charles University in Prague, Faculty of Mathematics and Physics, Institute of Particle and Nuclear Physics, V Holesovickach 2, CZ - 18000 Praha 8, Czech Republic 
${ }^{c b}$ Institute for High Energy Physics (IHEP), Federal Agency of Atom. Energy, Moscow Region, RU - 142 284 Protvino, Russia

${ }^{c c}$ Queen Mary, University of London, Mile End Road, E1 4NS, London, United Kingdom

${ }^{c d}$ Université Mohammed V, Faculté des Sciences, BP 1014, MO - Rabat, Morocco

${ }^{c e}$ Radboud University Nijmegen/NIKHEF, Dept. of Exp. High Energy Physics, Toernooiveld 1, NL - 6525 ED Nijmegen, Netherlands

${ }^{c f}$ Rutherford Appleton Laboratory, Science and Technology Facilities Council, Harwell Science and Innovation Campus, Didcot OX11 OQX, United Kingdom

${ }^{c g}$ University of Regina, Physics Department, Canada

${ }^{c h}$ Universidade Federal do Rio De Janeiro, Instituto de Fisica, Caixa Postal 68528, Ilha do Fundao, BR 21945-970 Rio de Janeiro, Brazil

${ }^{c i}$ Università La Sapienza, Dipartimento di Fisica and INFN Roma I, Piazzale A. Moro 2, IT-00185 Roma, Italy

${ }^{c j}$ Commissariat à l'Énergie Atomique (CEA), DSM/DAPNIA, Centre d'Etudes de Saclay, 91191 Gif-surYvette, France

${ }^{c k}$ Insitute of Physics, Academia Sinica, TW - Taipei 11529, Taiwan and Shandong University, School of Physics, Jinan, Shandong 250100, P. R. China

${ }^{c}$ University of Sheffield, Department of Physics \& Astronomy, Hounseld Road, Sheffield S3 7RH, United Kingdom

${ }^{c m}$ Insitute of Physics, Academia Sinica, TW - Taipei 11529, Taiwan

${ }^{c n}$ SLAC National Accelerator Laboratory, Stanford, California 94309, United States of America

${ }^{c o}$ University of South Carolina, Columbia, United States of America

${ }^{c p}$ Stockholm University, Department of Physics and The Oskar Klein Centre, SE - 10691 Stockholm, Sweden

${ }^{c q}$ Department of Physics and Astronomy, Stony Brook, NY 11794-3800, United States of America

${ }^{c r}$ Insitute of Physics, Academia Sinica, TW - Taipei 11529, Taiwan and Sun Yat-sen University, School of physics and engineering, Guangzhou 510275, P. R. China

${ }^{c s}$ Tbilisi State University, High Energy Physics Institute, University St. 9, GE - 380086 Tbilisi, Georgia

${ }^{c t}$ University of Toronto, Department of Physics, 60 Saint George Street, Toronto M5S 1A7, Ontario, Canada

${ }^{c u}$ University of Tsukuba, Institute of Pure and Applied Sciences, 1-1-1 Tennoudai, Tsukuba-shi, JP - Ibaraki 305-8571, Japan

${ }^{c v}$ University of California, Department of Physics \& Astronomy, Irvine, CA 92697-4575, United States of America

${ }^{c w}$ University College London, Department of Physics and Astronomy, Gower Street, London WC1E 6BT, United Kingdom

${ }^{c x}$ University of California Santa Cruz, Santa Cruz Institute for Particle Physics (SCIPP), Santa Cruz, CA 95064, United States of America

${ }^{c y}$ University of Illinois, Department of Physics, 1110 West Green Street, Urbana, Illinois 61801 United States of America

${ }^{c z}$ Instituto de Física Corpuscular (IFIC) Centro Mixto UVEG-CSIC Apdo. 22085 ES-46071 Valencia Dept. Física At. Mol. y Nuclear; Dept. Ing. Electrónica; Univ. of Valencia and Inst. de Microelectrónica de Barcelona (IMB-CNM-CSIC) 08193 Bellaterra Spain

${ }^{d a}$ University of Victoria, Department of Physics and Astronomy, P.O. Box 3055, Victoria B.C., V8W 3P6, Canada

${ }^{d b}$ University of Wisconsin, Department of Physics, 1150 University Avenue, WI 53706 Madison, Wisconsin, United States of America 
${ }^{d c}$ Bergische Universität, Fachbereich C, Physik, Postfach 100127, Gauss-Strasse 20, DE-42097 Wuppertal, Germany

dd Yale University, Department of Physics, PO Box 208121, New Haven, CT06520-8121, United States of America

de Yerevan Physics Institute, Alikhanian Brothers Street 2, AM - 375036 Yrevan, Armenia

E-mail: kjg@particle.kth.se

ABSTRACT: A new method for calibrating the hadron response of a segmented calorimeter is developed and successfully applied to beam test data. It is based on a principal component analysis of energy deposits in the calorimeter layers, exploiting longitudinal shower development information to improve the measured energy resolution. Corrections for invisible hadronic energy and energy lost in dead material in front of and between the calorimeters of the ATLAS experiment were calculated with simulated Geant4 Monte Carlo events and used to reconstruct the energy of pions impinging on the calorimeters during the 2004 Barrel Combined Beam Test at the CERN H8 area. For pion beams with energies between $20 \mathrm{GeV}$ and $180 \mathrm{GeV}$, the particle energy is reconstructed within $3 \%$ and the energy resolution is improved by between $11 \%$ and $25 \%$ compared to the resolution at the electromagnetic scale.

KEYwORDS: Calorimeter methods; Calorimeters; Detector modelling and simulations I; Pattern recognition, cluster finding, calibration and fitting methods 


\section{Contents}

1 Introduction $\quad 2$

2 The Layer Correlation method 3

3 The 2004 ATLAS Barrel Combined Beam Test 4

4 Calorimeter calibration to the electromagnetic scale 6

$\begin{array}{ll}4.1 \text { Cell energy reconstruction } & 6\end{array}$

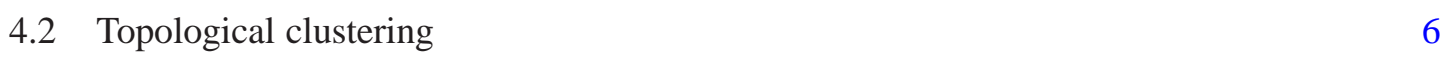

$\begin{array}{lll}4.3 & \text { Pion energy reconstruction } & 6\end{array}$

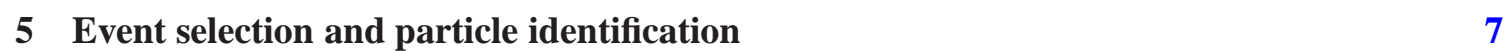

$\begin{array}{lll}5.1 & \text { Event selection } & 7\end{array}$

$\begin{array}{lll}5.2 & \text { Proton contamination } & 7\end{array}$

$6 \begin{array}{lll}6 & \text { Monte Carlo simulation } & 7\end{array}$

$\begin{array}{ll}6.1 & \text { Hadronic shower simulation }\end{array}$

6.2 Detector simulation 8

6.3 Event samples 8

7 Implementation of the Layer Correlation method 9

7.1 Calculation of the eigenvectors of the covariance matrix 9

$\begin{array}{ll}7.2 \text { Compensation weights } & 11\end{array}$

$\begin{array}{lll}7.3 & \text { Dead material corrections } & 12\end{array}$

7.3.1 Dead material between the LAr and Tile calorimeters 12

$\begin{array}{ll}\text { 7.3.2 Other dead material corrections } & 15\end{array}$

$\begin{array}{ll}7.4 & \text { Applying the calibration } \\ \end{array}$

8 Method validation on Monte Carlo simulation $\quad 17$

$\begin{array}{ll}8.1 \text { Compensation validation } & 17\end{array}$

$\begin{array}{ll}\text { 8.2 Dead material corrections } & 17\end{array}$

$\begin{array}{ll}\text { 8.3 Linearity and resolution in the Monte Carlo sample } & 17\end{array}$

9 Systematic uncertainties $\quad 20$

10 Application of the method to beam test data 20

10.1 Data to Monte Carlo simulation comparison 20

$\begin{array}{ll}10.2 \text { Linearity and resolution on data } & 22\end{array}$

11 Conclusions $\quad 25$ 


\section{Introduction}

In the general case of non-compensating calorimeters, the response to hadrons will be lower than the response to particles which only interact electromagnetically, such as electrons and photons. This is due to energy lost in hadronic showers in forms not measurable as an ionization signal, i.e., nuclear break-up, spallation and excitation, energy deposits arriving out of the sensitive time window (such as delayed photons), soft neutrons, and particles escaping the detector [1-3]. Moreover, the calorimeter response will be non-linear, since a hadronic shower has both an electromagnetic and a hadronic component, with the size of the former increasing with shower energy [4]. In addition, the large phase space of hadronic interactions leads to substantial fluctuations in the size of the electromagnetic shower component from event to event, degrading the measured energy resolution.

ATLAS [5] is one of the multi-purpose physics experiments at the CERN Large Hadron Collider (LHC) [6]. Scientific goals include searching for the Higgs boson and looking for phenomena beyond the Standard Model of particle physics, such as supersymmetry. Many measurements to be performed by the LHC experiments rely on a correct and accurate energy reconstruction of hadronic final-state particles. In the central barrel region, the ATLAS calorimeters consist of the lead-liquid argon (LAr) electromagnetic calorimeter and the Tile steel-scintillator hadronic calorimeter. Both calorimeters are intrinsically non-compensating.

Various techniques for equalizing the electromagnetic and hadronic shower response, i.e., achieving compensation, have been proposed. For a review, see [3], chapter 3. Software-based offline calibration techniques can use the topology of the visible deposited energy to exploit spatial event-by-event information on shower fluctuations and derive energy corrections aimed at restoring linearity in the response and improving the energy resolution. For example, the calorimeter cell energy density has been used for the calorimeter in the $\mathrm{H} 1$ experiment [7] and is planned to be used in ATLAS [8].

In this study, a calibration technique based on Monte Carlo simulation is developed to deal with compensating the response of a segmented calorimeter to hadrons and correcting for energy lost in the dead material between two calorimeter systems. The correlations between longitudinal energy deposits of the shower have been shown [9] to contain information on the electromagnetic and hadronic nature of the shower. This information is utilized by making a principal component analysis of the energies deposited in the different calorimeter layers. The calibration is applied to pion beam test data, taken at the 2004 ATLAS Barrel Combined Beam Test [10-14]. The method presented here is an alternative to the standard ATLAS calibration schemes. The application is quite specific to ATLAS, but the framework is general and it can be tested on any segmented calorimeter. Energy corrections based on the longitudinal shower development have been proposed by ATLAS in the context of jet calibration [15-17].

The following section explains the basic principles of the method. Section 3 details the ATLAS Barrel Combined Beam Test, while sections 4 and 5 discuss calibration to the electromagnetic scale 
and event selection, respectively. The Geant4 Monte Carlo simulation used is described in section 6. Then, section 7 gives the details of the implementation of the calibration method. In section 8 , the method is validated based on Monte Carlo simulations of pions. In the Monte Carlo simulation, the effect of the compensation weights and the dead material corrections are evaluated separately. Lastly, the linearity and resolution of the final calibrated energy is considered. Section 9 discusses systematic uncertainties. Results of applying the method to real beam test data are presented in section 10. Finally, conclusions are drawn in section 11.

\section{The Layer Correlation method}

The Layer Correlation calibration method (LC in the following) is aimed at calibrating the response of a non-compensating longitudinally segmented calorimeter to hadrons. Exploiting the properties of hadronic showers to characterize fluctuations in the deposited invisible energy, it uses a principal component analysis [18] of the energy deposited in the calorimeter layers. Observables that describe the shower fluctuations should be able to discriminate between different corrections to be applied to recover invisible losses due to hadronic interactions. Through the principal component analysis, it is possible to reduce the number of dimensions that the corrections depend on, while still capturing a large amount of event fluctuation information and maintaining a good separation between events with different content of invisible energy.

To derive the corrections, the interaction of the shower particles with the detector material is simulated with the Geant4 $[19,20]$ Monte Carlo simulation toolkit. In the simulation the true energy deposited in the calorimeters and the non-instrumented material is known. The covariance matrix between the calorimeter layer energy deposits is calculated. Diagonalizing it, a new orthogonal basis in the space of layer energy deposits is derived. It consists of the eigenvectors of the covariance matrix. By sorting the eigenvectors in descending eigenvalue order, the projection of the energy deposits in the calorimeter layers along the first few eigenvectors are made to describe the most important fluctuations in the longitudinal shower development.

Using this information, compensation weights-correcting for the non-compensation of the calorimeters - are derived in the form of two-dimensional look-up tables in the projections along the first two eigenvectors of the covariance matrix. One table is used for each calorimeter layer. The tables are thus functions of two different linear combinations of the observed energy deposits in the layers.

In addition, energy losses in non-instrumented material (so-called "dead material") will vary depending on the shower development. In the ATLAS barrel region, these losses are primarily in the region between the LAr and Tile calorimeters. The eigenvectors of the covariance matrix considered above can also be used to correct for this, resulting in a unified treatment for compensation and dead material correction by deriving both corrections from the same set of observables. In this implementation, the dead material corrections have an inherent dependence on the beam energy. This dependence is removed by employing an iteration scheme, where at each step the estimated energy of the former step is used, until the returned value is stable. A detailed mathematical description of the method is given in section 7 . 


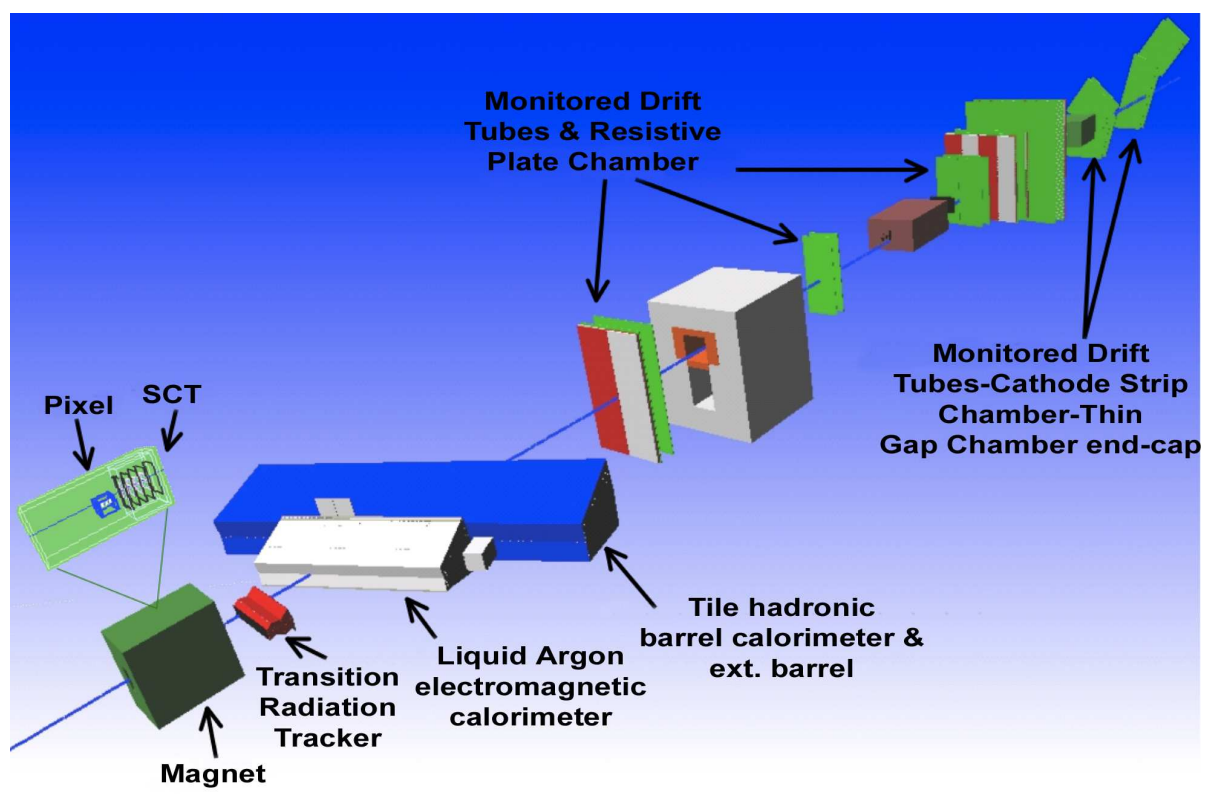

Figure 1. The layout of the 2004 Combined Beam Test.

\section{The 2004 ATLAS Barrel Combined Beam Test}

The energy calibration procedure is applied to data gathered in the fall of 2004 during the ATLAS Barrel Combined Beam Test at the H8 beam line of the CERN SPS accelerator. A full slice of the ATLAS barrel region was installed (see Figure 1). This included, firstly, the inner tracker with the pixel detector, the silicon strip semiconductor tracker (SCT), and the straw tube transition radiation tracker (TRT); secondly, the LAr and Tile calorimeters; and thirdly, the muon spectrometer. The pixel and SCT detectors were surrounded by a magnet capable of producing a field of $2 \mathrm{~T}$, although no magnetic field was applied in the runs used for this study.

The pixel detector [5] comprises six modules, each consisting of a single silicon wafer with an array of $40 \times 400 \mu \mathrm{m}^{2}$ pixels. The modules were arranged in locations mimicking the ATLAS configuration, with an approximate angle of 20 degrees with respect to the incoming beam. The semiconductor tracker (SCT) [5] uses sets of stereo strips for tracking. Each module gives two hits, one in each direction. Eight modules, corresponding to those in the ATLAS end-cap, were used. The TRT [5] forms the outermost tracking system in ATLAS. It consists of a collection of $4 \mathrm{~mm}$ diameter polyimide straw tubes filled with a mixture of xenon, carbon dioxide, and oxygen [5]. Transition radiation is emitted when a charged particle crosses the interface between two media having different refractive index. The amount of emitted radiation depends on the Lorentz $\gamma$ factor of the particle. This makes it possible to discriminate between electrons and hadrons, given the much higher $\gamma$ factor of the former at a given energy, due to their smaller mass.

Details of the ATLAS LAr electromagnetic calorimeter are described elsewhere [5, 21]. In the beam test one calorimeter module was used. The calorimeter is made from $2.21 \mathrm{~mm}$ thick 


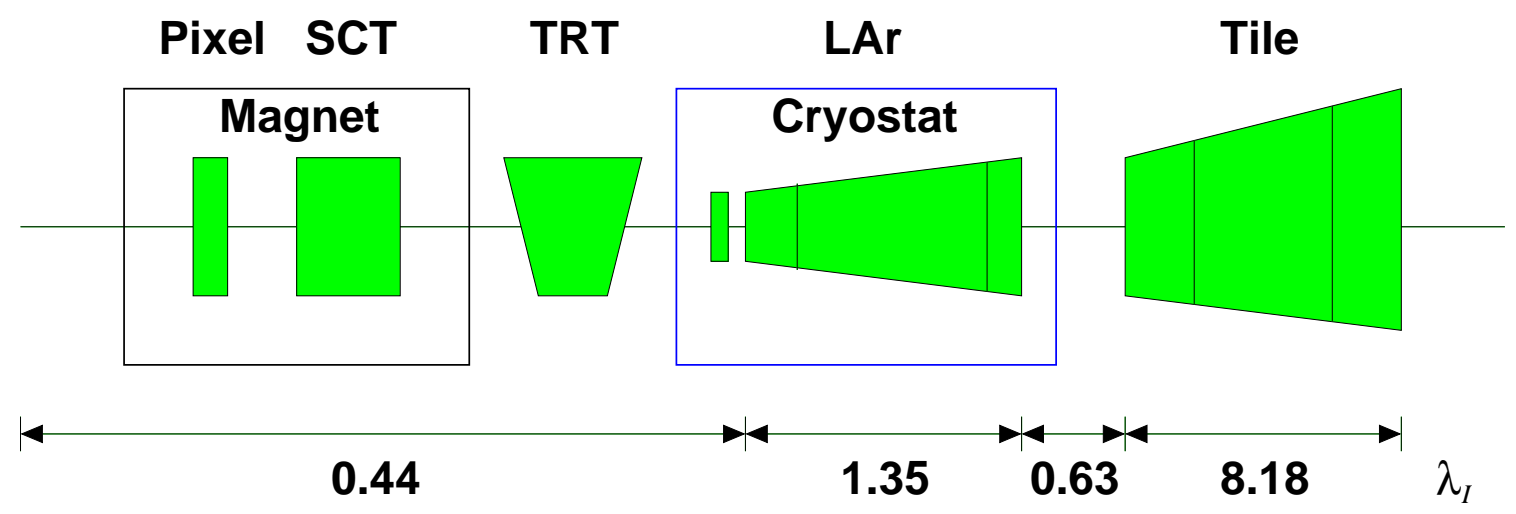

Figure 2. The layout of the 2004 Combined Beam Test.

accordion-shaped lead absorbers glued between stainless steel cathodes. Three-layered anode electrodes are interleaved between the absorbers, spaced by $2 \mathrm{~mm}$ gaps over which a high voltage of $2 \mathrm{kV}$ is applied. The module was placed in a cryostat containing liquid argon. The signal is read out by capacitive coupling between the two outermost and the central layer of the anodes. In front of this accordion module a thin presampler module was mounted. It consists of two straight sectors with alternating cathode and anode electrodes glued between plates made of a fiber-glass epoxy composite (FR4). The Tile hadronic calorimeter consists of iron absorbers sandwiched between organic scintillator tiles. It is described in detail elsewhere [5, 22]. The tiles and absorbers are oriented parallel to the direction of incoming particles. Every cell of the calorimeter is read out by two wavelength-shifting fibers, which in turn are grouped together and read out by photo-multiplier tubes (PMTs).

The calorimeters were placed so that the beam impact angle corresponded to a pseudo-rapidity ${ }^{1}$ of $\eta=0.45$ in the ATLAS detector. At this angle, the expected amount of material in front of the calorimeters was about $0.44 \lambda_{I}$, where $\lambda_{I}$ is the nuclear interaction length $[3,23]$. This includes the LAr presampler. The LAr calorimeter proper is longitudinally segmented in three layers that extend in total for $1.35 \lambda_{I}$. The dead material between the LAr and Tile calorimeters spans about $0.63 \lambda_{I}$. Finally the three longitudinal segments of the Tile calorimeter stretched in total for about $8.18 \lambda_{I}$. A sketch of this setup is shown in Figure 2. In total there are seven longitudinal calorimeter layers (the LAr presampler; the front, middle, and back layers of the LAr calorimeter; and the so-called $\mathrm{A}, \mathrm{BC}$, and D layers of the Tile calorimeter). The length of the individual calorimeter layers was 0.32, 0.96, and $0.07 \lambda_{I}$ in the LAr calorimeter and 1.61, 4.53, and 2.04 $\lambda_{I}$ in the Tile calorimeter.

In addition, special beam-line detectors were installed to monitor the beam position and reject background events. Those include beam chambers monitoring the beam position and trigger scintillators. Beams consisting of electrons, photons, pions, protons, and muons were studied. In this analysis, pion beams with nominal momenta of 20, 50, 100, and $180 \mathrm{GeV}$ were used (see Table 1). Data belong to the fully combined run period, where all detector sub-systems were present

\footnotetext{
${ }^{1}$ ATLAS has a coordinate system centered on the interaction point, with the $x$ axis pointing towards the center of the LHC ring, the $y$ axis pointing straight up, and the $z$ axis parallel to the beam. Pseudo-rapidity is defined as $-\ln (\tan (\theta / 2))$, where $\theta$ is the angle to the positive $z$ axis.
} 
and operational. No magnetic field was applied around the pixel and silicon strip detectors. The beams were produced by letting $400 \mathrm{GeV}$ protons from the SPS accelerator impinge on a beryllium target, from which secondary pions are selected. For the run at $180 \mathrm{GeV}$, positrons were nominally selected after the target. However, the beam still contained a contamination of positively charged pions, which were selected and used for this analysis with the methods described in section 5.1.

\section{Calorimeter calibration to the electromagnetic scale}

\subsection{Cell energy reconstruction}

The individual cells of the calorimeter are calibrated to the electromagnetic scale, i.e., with the aim of correctly measuring the energy deposited in the cell by a purely electromagnetic shower. The calibration of electronics of the LAr calorimeter is described in detail in [24]. The method of optimal filtering [25] is used to reconstruct the amplitude of the shaped signal, which is sampled by an ADC (analog-to-digital converter) at $40 \mathrm{MHz}$. The amplitude is calculated as weighted sum of the samples, after a pedestal level measured using random triggers is subtracted. $F_{\mu \mathrm{A} \rightarrow \mathrm{MeV}} / f_{\text {samp }}$, a constant factor, converts the measured current to an energy measured in MeV. The energy deposited in the lead absorbers is taken into account by the sampling fraction $f_{\text {samp }}$. The shaping electronics is calibrated by inserting calibration pulses of known amplitude. In the Tile calorimeter a parameterized pulse shape is fitted to the samples. A charge injection system is used to calibrate the read-out electronics, while a cesium source is used to equalize the cell response, including the response of the PMTs (see, for example [26]).

\subsection{Topological clustering}

Calorimeter cells calibrated to the electromagnetic scale are combined by adding up the energy in neighboring cells using a topological cluster algorithm [27]. The algorithm has three adjustable thresholds: Seed $(S)$, Neighbor $(N)$, and Boundary $(B)$. First, seed cells having an energy above the $S$ threshold are found and a cluster is formed starting with this cell. Then, neighboring cells having an energy above the $N$ threshold are added to the cluster. This process is repeated until the cluster has no neighbors with an energy above the $N$ threshold. Finally, all neighboring cells having an energy above the $B$ threshold are added to the cluster. To avoid bias, the absolute values of the cell energies are used. The $S, N$, and $B$ thresholds are set to, respectively, four, two, and zero times the expected noise standard deviation in the cell considered.

\subsection{Pion energy reconstruction}

The reconstructed energy in a calorimeter layer $L$ is obtained by considering all the topological clusters in the event and summing up the parts of the clusters that are part of that calorimeter layer. The total reconstructed energy is then derived by summing over the $N_{\text {lay }}$ longitudinal layers in the calorimeter. 


\begin{tabular}{|c|c|c|c|c|}
\hline$E_{\text {beam }}^{\text {nom }}(\mathrm{GeV})$ & $E_{\text {meas }}(\mathrm{GeV})$ & No. ev. bef. cuts & No. ev. after cuts & $f_{\text {prot }}$ \\
\hline \hline 20 & 20.16 & 49871 & 8957 & $<17 \%(84 \% \mathrm{CL})$ \\
50 & 50.29 & 109198 & 29578 & $(45 \pm 12) \%$ \\
100 & 99.89 & 67220 & 5843 & $(61 \pm 6) \%$ \\
180 & 179.68 & 105082 & 11780 & $(76 \pm 4) \%$ \\
\hline
\end{tabular}

Table 1. Data samples taken in the 2004 Combined Beam Test used in the present analysis.

\section{Event selection and particle identification}

\subsection{Event selection}

A signal in the trigger scintillator and a measurement in adjacent beam chambers that is compatible with one particle passing close to the nominal beam line are required. In addition, exactly one track, where the sum of the number of hits in the Pixel detector and the SCT is more than six, is asked for, as well as at least 20 hits in the TRT. The track in the TRT must be compatible with a pion track, i.e., no more than two hits passing the high threshold must be present. Events with a second track in the TRT are rejected: this ensures that the pion does not interact strongly before the TRT. Furthermore, there must be at least one topological cluster (see section 4.2) with at least $5 \mathrm{GeV}$ in the calorimeter. This cut rejects muons contained in the beam and does not influence the pion energy measurement. To reject some residual electron background, events with more than $99 \%$ of their energy in the LAr calorimeter are excluded. The same selection is applied on simulated Monte Carlo events as on data, with the exception of cuts related to the beam chambers and scintillators.

\subsection{Proton contamination}

This study used beams of pions with positive electric charge. These beams are known to have a sizable proton contamination $f_{\text {prot }}$ defined as the fraction of events in a sample that result from protons impinging on the calorimeters. It varies between different beam energies. The TRT makes it possible to measure the average proton contamination of the test beam for each beam energy, owing to the different probabilities between pions and protons of emitting transition radiation, although it is not possible to discriminate between the particles on an event-by-event basis. The measured [10] contamination is reported in Table 1. For the $20 \mathrm{GeV}$ beam energy, a one-sided confidence interval is given. In the analysis, a proton contamination of $0 \%$ was used. Agreement is found with measurements performed by a Čerenkov counter at a 2002 beam test [28] conducted in the same beam line.

\section{Monte Carlo simulation}

\subsection{Hadronic shower simulation}

All calibration corrections are extracted from a Geant4.7 [19, 20] Monte Carlo simulation, with an accurate description of the Combined Beam Test geometry. The physics list—i.e., set of modelsQGSP_BERT was used. It uses the QGSP [29] (Quark Gluon String Pre-compound) phenomenological model describing the hadron-nucleus interaction by the formation and fragmentation of 
excited strings together with the de-excitation of an excited nucleus. The Bertini model [30-32] of the intra-nuclear hadronic cascade is used to describe nuclear interactions at low energies. This model treats the particles in the cascade as classical and propagates them through the nucleus, which is modeled as a medium with a density averaged in concentric spheres. Excited states are collected and the nucleus decays in a slower phase following the fast intra-nuclear cascade.

The Bertini model is applied up to an energy of $9.9 \mathrm{GeV}$, while the QGSP model applies from $12 \mathrm{GeV}$ and upward. In an intermediate range of $9.5-25 \mathrm{GeV}$, the low-energy parameterized LEP model [33] is used. In the energy ranges where models overlap, the decision which one to use is made stochastically using a continuous linear probability distribution that goes from exclusively using the low-energy model at the lower end of the region to exclusively using the high-energy model at the upper end.

\subsection{Detector simulation}

The simulation provides not only reconstructed calorimeter cell energies at the electromagnetic scale—including the effects of the readout electronics—but also the true deposited energy, which is divided into four components: electromagnetic visible, hadronic visible, invisible, and escaped. Visible energy results from ionization of the calorimeter material. Invisible energy is energy not directly measurable in the detector, such as break-up energy in nuclear interactions. The escaped energy represents the small contribution from neutrinos, high-energy muons and, possibly, neutrons and low-energy photons escaping the total simulated volume.

\subsection{Event samples}

Monte Carlo samples were produced by simulating both pions and protons impinging on the detector setup. Two statistically independent event samples were produced by dividing the available sample into two approximately equal parts: one set ("correction" samples in the following) was used to derive compensation weights and dead material corrections, while the other set ("signal" samples in the following) was used to validate the weighting procedure and find the expected performance. Pions and protons were simulated at 25 different beam energies, ranging from $15 \mathrm{GeV}$ to $230 \mathrm{GeV}$. In total, about 800000 events per sample and particle type were available after event selection. The energy spacing was 2,3 , or $5 \mathrm{GeV}$ up to $70 \mathrm{GeV}$ and 10 or $20 \mathrm{GeV}$ above $70 \mathrm{GeV}$. This spacing was found to give satisfactory performance (see sections 8 and 10). Further studies of different spacings can be pursued when applying this technique to different calorimeters to explore possible improvement in performance.

Taking the proton beam contamination mentioned in section 5.2 into account, all the available "correction" Monte Carlo samples were used to build a "mixed" pion-proton sample, one for each energy available in the data (see Table 1). Each of these samples is used as input when deriving the corrections used for that proton fraction. In this way the corrections were tuned to the studied proton fraction. If the samples had different numbers of events, a sample-dependent weight was first applied to give them equal weight before selection cuts. Then, given the proton contamination $f_{\text {prot }}$ at a given energy, pion and proton events for each same-energy pair of samples were assigned a weight of $1-f_{\text {prot }}$ and $f_{\text {prot }}$, respectively. 


\section{Implementation of the Layer Correlation method}

\subsection{Calculation of the eigenvectors of the covariance matrix}

Each event is associated with a set of $N_{\text {lay }}$ layer energy deposits $\left(E_{1}^{\text {rec }}, \ldots, E_{N_{\text {lay }}}^{\text {rec }}\right)$, one per calorimeter layer, representing a point in an $N_{\text {lay }}$-dimensional vector space, referred to in the following as the space of layer energy deposits. They are reconstructed energies at the electromagnetic scale, formed as calorimeter layer sums of topological clusters as described in section 4.1. The $N_{\text {lay }}{ }^{-}$ dimensional covariance matrix of the layer energy deposits is calculated as

$$
\operatorname{Cov}(M, L)=\left\langle E_{M}^{\mathrm{rec}} E_{L}^{\mathrm{rec}}\right\rangle-\left\langle E_{M}^{\mathrm{rec}}\right\rangle\left\langle E_{L}^{\mathrm{rec}}\right\rangle
$$

where $M$ and $L$ denote calorimeter layers and $E_{M}^{\text {rec }}$ is the energy reconstructed at the electromagnetic scale in calorimeter layer $M$. The averages are defined as

$$
\left\langle E_{M}^{\mathrm{rec}} E_{L}^{\mathrm{rec}}\right\rangle=\frac{\sum_{i} E_{M, i}^{\mathrm{rec}} E_{L, i}^{\mathrm{rec}}}{N_{\mathrm{ev}}} \text { and }\left\langle E_{M}^{\mathrm{rec}}\right\rangle=\frac{\sum_{i} E_{M, i}^{\mathrm{rec}}}{N_{\mathrm{ev}}}
$$

The sums are performed over all the $N_{\mathrm{ev}}$ events in the sample. The eigenvectors of the covariance matrix form a new orthogonal basis in the space of layer energy deposits. The coordinates of the point in the $N_{\text {lay }}$-dimensional vector space corresponding to an event $i$ can be expressed in this new eigenvector basis as

$$
E_{\mathrm{eig}, M}^{\mathrm{rec}}=\sum_{L} \alpha_{M, L}^{\mathrm{rec}} E_{L}^{\mathrm{rec}},
$$

where $\alpha_{M, L}^{\mathrm{rec}}$ are the coefficients of the transition matrix to the new basis. Projections of events along the covariance matrix eigenvectors represent independent fluctuations. The variances of those fluctuations are given by the corresponding eigenvalues. The eigenvectors are sorted in descending order according to their eigenvalues, meaning that the first eigenvectors determine the directions along which most of the event fluctuations take place. The layer energy covariance matrix $\operatorname{Cov}(M, L)$ (equations 7.1 and 7.2) is calculated using events from the "mixed" sample.

In any given event a symmetric energy cut is applied on each layer energy such that the energy for that layer is re-defined as $E_{L}^{\text {rec }}$, if $\left.\left|E_{L}^{\text {rec }}\right|\right\rangle E_{L}^{\text {thr }}$, zero otherwise. The goal of such cuts is to eliminate the contribution of noise-dominated layers. The energy threshold values for each calorimeter layer can be found in Table 2. The cuts were optimized to obtain the best expected compensation performance on Monte Carlo samples at $50 \mathrm{GeV}$.

A physical interpretation of the eigenvalues and normalized eigenvectors can be obtained from Figure 3, which shows the components of the first three eigenvectors expressed in the original basis of calorimeter layer energy deposits. We find that

$$
\begin{aligned}
& E_{\mathrm{eig}, 0}^{\mathrm{rec}} \approx \frac{1}{\sqrt{6}}\left(-2 E_{\mathrm{LAr}, \text { middle }}+E_{\mathrm{Tile}, \mathrm{A}}+E_{\mathrm{Tile}, \mathrm{BC}}\right), \\
& E_{\mathrm{eig}, 1}^{\mathrm{rec}} \approx \frac{1}{\sqrt{2}}\left(-E_{\mathrm{Tile}, \mathrm{A}}+E_{\mathrm{Tile}, \mathrm{BC}}\right), \text { and } \\
& E_{\mathrm{eig}, 2}^{\mathrm{rec}} \approx \frac{1}{\sqrt{3}}\left(E_{\mathrm{LAr} \text {,middle }}+E_{\mathrm{Tile}, \mathrm{A}}+E_{\mathrm{Tile}, \mathrm{BC}}\right) .
\end{aligned}
$$




\begin{tabular}{|c|c|}
\hline Calorimeter layer & Threshold $(\mathrm{GeV})$ \\
\hline \hline 0 & 0.032 \\
1 & 0.108 \\
2 & 0.030 \\
3 & 0.150 \\
4 & 0.039 \\
5 & 0.070 \\
6 & 0.042 \\
\hline
\end{tabular}

Table 2. Energy thresholds per calorimeter layer.
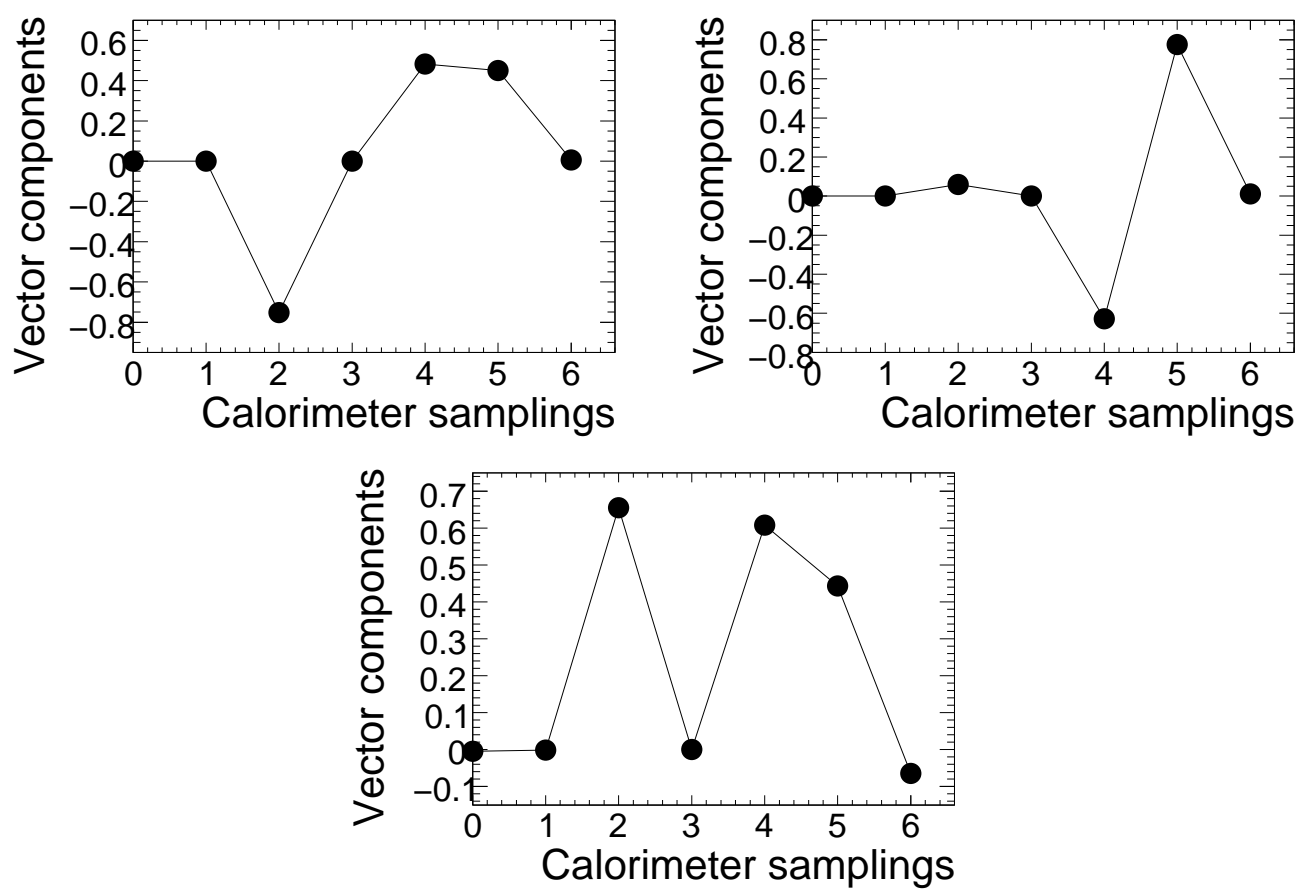

Figure 3. Eigenvector components for the first three eigenvectors expressed in the basis of the seven layers of the ATLAS calorimeters in the Combined Beam Test for a simulated mix of protons and pions with $45 \%$ proton contamination.

So in a qualitative but suggestive way, we can make the interpretation that $E_{\text {eig, } 0}^{\mathrm{rec}}$ corresponds to the difference between the Tile and LAr calorimeters, since most of the energy deposited in the LAr calorimeter is deposited in the middle layer. $E_{\text {eig, } 1}^{\mathrm{rec}}$ corresponds to the difference between the second and first layers of the Tile calorimeter, while $E_{\mathrm{eig}, 2}^{\mathrm{rec}}$ corresponds to most of the energy of the event. The other eigenvectors represent individual calorimeter layers. These layers are rather thin and appear to be uncorrelated to the other layers. 


\subsection{Compensation weights}

The compensation weights account for the non-linear response of the calorimeters to hadrons. There is one weight table for each calorimeter layer, i.e., three for the LAr calorimeter and three for the Tile calorimeter. The seventh layer, the LAr presampler, which in order is the first layer, is not used in the weighting procedure, as explained below. The total reconstructed energy is the sum of the weighted energies in each calorimeter layer:

$$
\begin{aligned}
E_{L}^{\text {weighted }} & =w_{L} E_{L}^{\mathrm{rec}} \\
E_{\text {tot }}^{\text {weighted }} & =\sum_{L} E_{L}^{\text {weighted }} .
\end{aligned}
$$

For each event $i$, there is an ideal set of $N_{\text {lay }}$ coefficients that would re-weight each reconstructed energy deposit in layer $L$ to the true deposited energy:

$$
w_{L, i}^{\text {ideal }}=E_{L, i}^{\text {true }} / E_{L, i}^{\text {rec }}
$$

The symbol $E_{L, i}^{\text {rec }}\left(E_{L, i}^{\text {true }}\right)$ denotes the reconstructed (true) energy deposited in the $L^{\text {th }}$ layer in the $i^{\text {th }}$ event. The task is to find a set of weights $w_{L}$ that approximate the ideal weights. In general, for

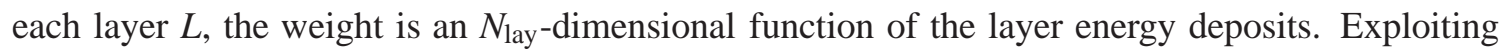
the fluctuation-capturing properties of the eigenvector projections, the weights can in general be derived as a function of an $N$-dimensional subspace of the $N_{\text {lay }}$-dimensional space of layer energy deposits, spanned by the first $N$ eigenvectors. In the absence of an analytic formulation, the layer weights $w_{L}$ are estimated by Monte Carlo sampling: multi-dimensional cells are built, which partition the $N$-dimensional vector space along the directions of the base eigenvectors. In general, these cells are multi-dimensional hyper-cubes. They are referred to as bins below.

For each bin $k$ one defines the weight as the average of the ideal weights of equation 7.9:

$$
w_{k, L}=\left\langle E_{L, i}^{\mathrm{true}} / E_{L, i}^{\mathrm{rec}}\right\rangle_{k}=\frac{1}{N_{\mathrm{ev}, k}} \sum_{i} E_{L, i}^{\mathrm{true}} / E_{L, i}^{\mathrm{rec}}
$$

where the summation is performed for the $N_{\mathrm{ev}, k}$ events in the bin. If each event has a weight ${ }^{2} p_{i}$, the average is modified accordingly:

$$
w_{k, L}=\left\langle E_{L, i}^{\mathrm{true}} / E_{L, i}^{\mathrm{rec}}\right\rangle_{k}=\frac{\sum_{i} p_{i} E_{L, i}^{\mathrm{true}} / E_{L, i}^{\mathrm{rec}}}{\sum_{i} p_{i}}
$$

Using bin $k$ of the weight tables, the total reconstructed energy becomes

$$
E_{\mathrm{tot}, k}^{\mathrm{weighted}}=\sum_{L} w_{k, L} E_{L}^{\mathrm{rec}} .
$$

Here, the $w_{k, L}$ functions defined in equation 7.11 are estimated in bins of the two-dimensional space spanned by the eigenvectors corresponding to the two highest eigenvalues, i.e., $N=2$. Thus each layer is associated with a two-dimensional look-up table. For a given layer the average weights in each two-dimensional bin are calculated using only the energy values that passed the cuts defined in section 7.1. The table has the same number of equally spaced bins along the two dimensions:

\footnotetext{
${ }^{2}$ For instance, to equalize the number of events for all data sets.
} 
$128 \times 128$. Bi-linear interpolation is performed between the bins. Weights for the LAr presampler are not calculated, even if the presampler is kept in the covariance matrix. No weights are applied to the energy deposited in the presampler layer, and energy deposited in the presampler itself is taken as part of the upstream dead material losses.

In addition the compensation weights and corrections derived from the proton sample are corrected by the factor

$$
\frac{E_{\text {beam }}^{\text {nom }}}{E_{\text {beam }}^{\text {nom }}-m_{\text {proton }}}
$$

where $m_{\text {proton }}$ is the proton mass, to account for the fact that, for a proton, the sum of the total true deposited energy in the calorimeter is $E_{\text {beam }}^{\text {nom }}-m_{\text {proton }}$.

Typical compensation weight tables are shown in Figure 4: they illustrate the look-up tables for the second (middle) layer of the LAr calorimeter and for the first and second layer of the Tile calorimeter for a pion-proton mixed sample with $45 \%$ contamination. The triangular shape visible in the weight tables can be understood from the interpretation of the eigenvectors of equations 7.4 and 7.5. With increasing energy in the Tile calorimeter and less in the LAr calorimeter, i.e., $E_{\text {eig, } 0}^{\mathrm{rec}}$ is large, there are more values that can be assumed by $E_{\text {eig, }, 1}^{\mathrm{rec}}$, which is the approximate difference between the first and second layers of the Tile calorimeter. Three lines can be seen extending from the origin to each of the three corners of the triangle. Firstly, the line extending from the origin and to the left corresponds to events where close to all of the energy is deposited in the LAr calorimeter. The small slope is due to the slight dependence of $E_{\text {eig, } 1}^{\mathrm{rec}}$ on the second layer of the LAr calorimeter. Secondly, the line extending up and to the right corresponds to events where all energy is deposited in the second layer of the Tile calorimeter. Along that line, weights are small for the first sampling of the Tile calorimeter, since particles are still minimum-ionizing in that layer. Thirdly the faint line extending down and to the right corresponds to events where close to all the energy is deposited in the first layer of the Tile calorimeter.

\subsection{Dead material corrections}

Regions of dead material constitute those parts of the experiment that are neither active calorimeter read-out material (liquid argon or scintillator), nor sampling calorimeter absorbers (mostly lead or steel). The LC technique is used for the dead material between the LAr and the Tile calorimeters, while a simple parameterized model is utilized for other losses.

\subsubsection{Dead material between the LAr and Tile calorimeters}

Most of the dead material is in the LAr cryostat wall between the LAr and Tile calorimeters. In this $0.6 \lambda_{I}$ region, pion showers are often fully developed, giving rise to large energy loss. Each event $i$ is associated with a point in the layer energy deposit vector space as explained in section 7.1. It also has a true total energy lost in the dead material between the LAr and Tile calorimeters: $E_{\mathrm{LArTile}}^{\mathrm{DM}, \mathrm{true}}(i)$. The dead material correction $E_{\mathrm{LArTile}}^{\mathrm{DM}}$ for each event $i$ can be derived as a $T$-dimensional function of the layer energy deposits. In general, the subspace chosen for deriving the dead material correction and its dimension $T$ can be different from the one chosen for compensation, both in content (spanned by different eigenvectors) and in dimension ( $T$ can be different from $N$ ). The 


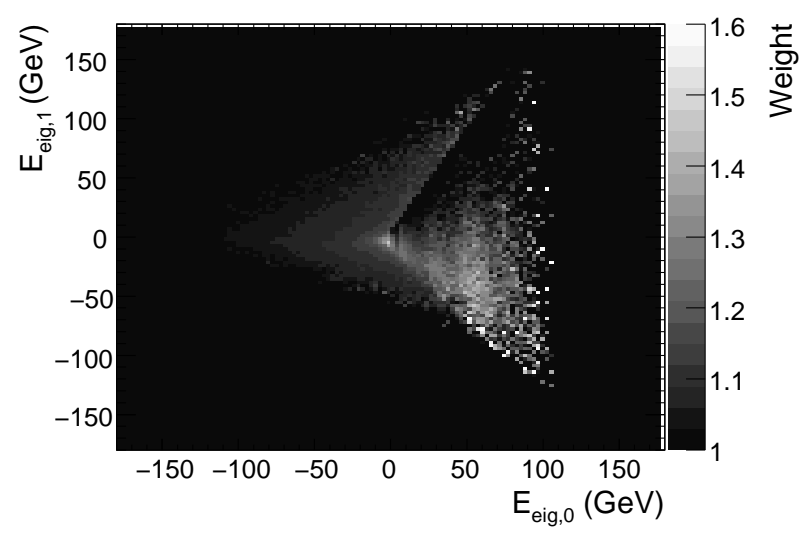

(a)

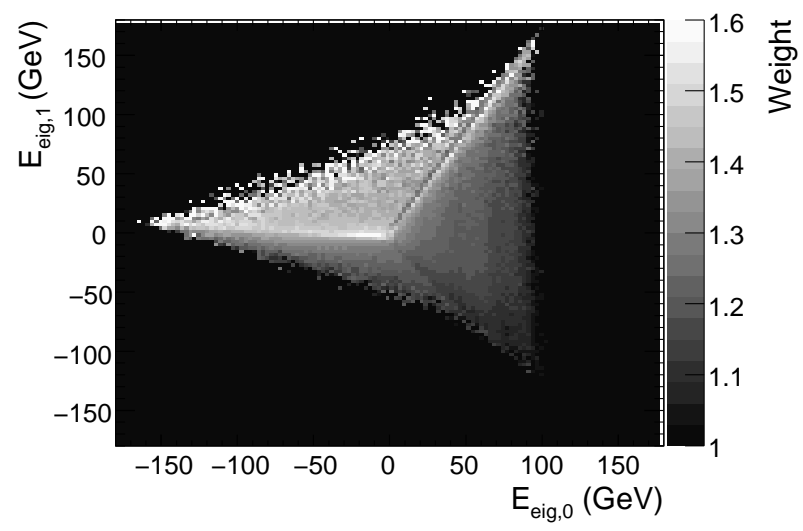

(b)

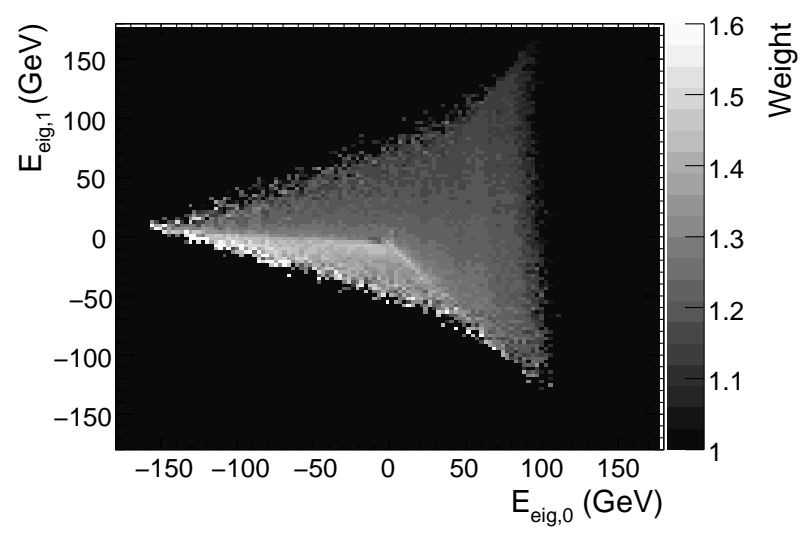

(c)

Figure 4. Compensation weights as a function of the first two eigenvector projections for simulated pionproton mixed events (45\% proton contamination) in the second layer of the LAr calorimeter (a), first layer of the Tile calorimeter (b), and second layer of the Tile calorimeter (c). 


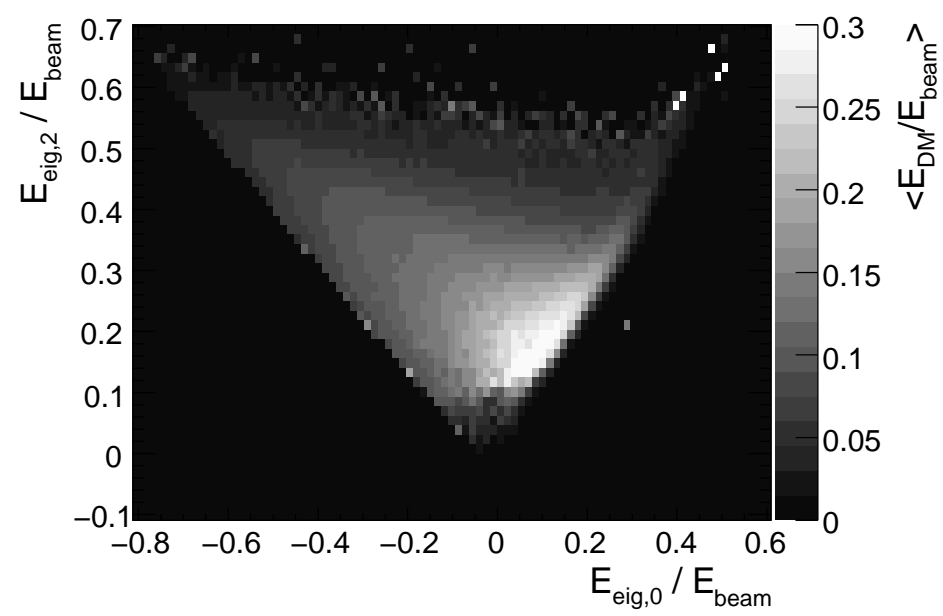

Figure 5. Look-up table for LAr-Tile dead material corrections as a function of the first and third eigenvector projections normalized to beam energy for $45 \%$ proton contamination.

value of $E_{\mathrm{LArTile}}^{\mathrm{DM}}$ is estimated by Monte Carlo sampling. For any $T$-dimensional bin $m$ one defines

$$
E_{\mathrm{LArTile}, m}^{\mathrm{DM}}=\left\langle E_{\mathrm{LArTile}, i}^{\mathrm{DM},\rangle_{m},}\right.
$$

where the average is performed for the events in that bin.

Here, the correction defined in equation 7.14 is calculated in bins of the two-dimensional space spanned by the eigenvectors corresponding to the first and third eigenvalues, i.e., $T=2$. This was the combination of eigenvectors that was found to give the best performance. As for the compensation weights, correction tables are derived from a $128 \times 128$ bin look-up table and bi-linear interpolation is performed between the bins.

The three dimensions of the look-up table are all shown to scale with the beam energy, i.e., a table determined at a given beam energy can be turned into one at a different beam energy by scaling all the dimensions with the ratio of the two energies. Consequently, all dimensions in the table - the eigenvector projections and the average dead material losses-are divided by the beam energy when filling the table. That is, the event coordinates in the space of layer energy deposits are expressed as

$$
E_{\mathrm{eig}, M}^{\mathrm{rec}, \mathrm{norm}}=E_{\mathrm{eig}, M}^{\mathrm{rec}} / E=\sum_{L} \alpha_{\mathrm{eig}, L}^{\mathrm{rec}} E_{L}^{\mathrm{rec}} / E
$$

where the variables have the same meaning as in equation 7.3 and $E$ is the best estimate of the beam energy of the simulated pion in that event (see below). The dead material look-up table is shown in Figure 5 for a pion-proton mixed sample with $45 \%$ contamination. The figure shows the distribution of the rescaled dead material energy as a function of the rescaled event coordinates. Regions with different dead material fractions can be differentiated. They range between 0 and more than $30 \%$ of beam energy. In addition, the samples at different energies behave very similarly as a function of the re-scaled variables. 


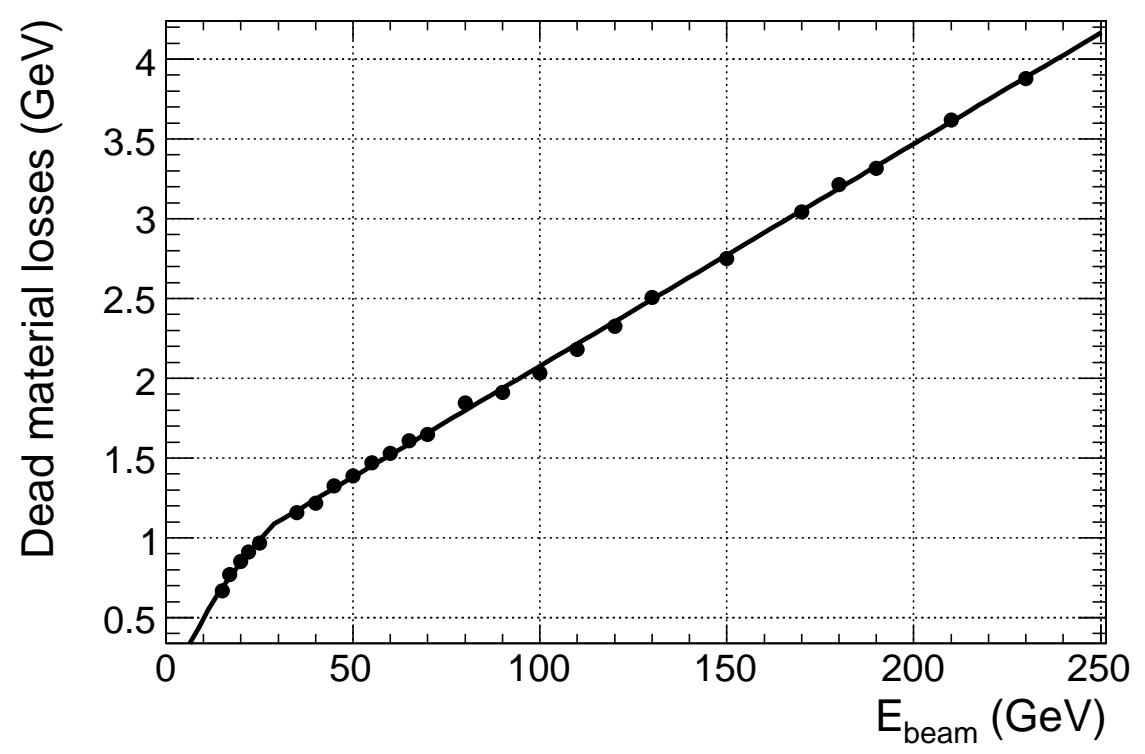

Figure 6. Mean dead material losses other than those between the LAr and Tile calorimeters as a function of the beam energy. Filled circles indicate the mean loss obtained from Monte Carlo simulation. The line indicates a parameterization to interpolate between the beam energies.

\subsubsection{Other dead material corrections}

While the energy losses between the LAr and Tile calorimeters dominate, there are still other regions where dead material losses can occur. These are losses located in the material upstream of the LAr calorimeter, between the LAr presampler and the first LAr calorimeter layer, and energy leakage beyond the Tile calorimeter. To compensate for these losses the mean energy loss was determined as a function of beam energy and the resulting data points were fitted using a suitable functional form

$$
E_{\text {other }}^{\mathrm{DM}}\left(E_{\text {beam }}\right)=\left\{\begin{aligned}
C_{1}+C_{2} \sqrt{E_{\text {beam }}} & \text { if } E_{\text {beam }}<E_{0} \\
C_{3}+C_{4}\left(E_{\text {beam }}-E_{0}\right) & \text { otherwise, }
\end{aligned}\right.
$$

where $E_{0}=30 \mathrm{GeV}$. As an example, the fit for a proton fraction of $45 \%$ can be seen in Figure 6 . The resulting fitted parameters are

$$
\begin{aligned}
& C_{1}=(-353 \pm 23) \mathrm{MeV}, \\
& C_{2}=(8.47 \pm 0.17) \sqrt{\mathrm{MeV}}, \\
& C_{3}=(1102 \pm 3) \mathrm{MeV}, \quad \text { and } \\
& C_{4}=0.01392 \pm 0.0001 .
\end{aligned}
$$




\subsection{Applying the calibration}

The final energy after calibration consists of the sum of the weighted calorimeter layer energies and the dead material corrections:

$$
E_{\mathrm{tot}, k, m}^{\mathrm{corr}}=E_{\mathrm{tot}, k}^{\text {weighted }}+E_{\mathrm{tot}, m}^{\mathrm{DM}}(E) .
$$

The index $k$ stands for the bin in the appropriate $N$-dimensional space of layer energy deposits used in the weight tables (equations 7.10 or 7.11), while $m$ is the bin in the $T$-dimensional space of layer energy deposits used to build the LC estimate for the energy loss in the dead material between the LAr and Tile calorimeters obtained from equation 7.14. The total dead material correction is derived from summing the two contributions derived in sections 7.3.1 and 7.3.2:

$$
E_{\mathrm{tot}, m}^{\mathrm{DM}}(E)=E_{\mathrm{LArTile}, m}^{\mathrm{DM}}+E_{\mathrm{other}}^{\mathrm{DM}}(E),
$$

where $E$ is the best estimate for the total deposited pion energy used to estimate $E_{\text {beam }}$ in equation 7.16.

The events in a Monte Carlo sample are usually generated at a fixed beam energy in order to test the calorimeter response. Corrections derived from a fixed beam energy sample are, in principle, dependent on that information, i.e., they depend on the same quantity (pion energy) for the reconstruction of which they should be used. For the compensation weights, this dependence is overcome by superposing events from all the available energies. The eigenvector projections scale approximately with the energy of the incoming particle, meaning that regions in the table that come in use for a certain particle energy will be dominated by samples close to that energy.

On the other hand, the look-up-table-based LAr-Tile dead material correction and the parameterized model for the other dead material losses have an inherent dependence on an assumed beam energy when applying the corrections (see equations 7.15 and 7.22). This dependence is overcome using an iteration technique, giving the end result of depending only on the energy in the calorimeters. At each step the best estimate of the reconstructed energy $E_{\text {tot }}^{\text {corr }}$ after all corrections is used to set both the scaling factor $1 / E$ (equation 7.15) for the LAr-Tile correction and the best pion energy estimate in the parameterization for the other dead material corrections. Each new estimate of the energy is used to pick up a new correction from the look-up table until the returned value is stable. In the initial step $E_{\text {tot }}^{\text {corr }}$ is just the pion energy after compensation weights are applied. The iteration cut-off is a tunable parameter.

The process of applying the calibration is as follows:

- Associate each event to a bin in both the $N$-dimensional compensation weight and the $T$ dimensional dead material correction spaces defined in sections 7.2 and 7.3 by expressing its electromagnetic-scale energy deposit vector in the new eigenvector basis derived from the simulated events.

- Extract compensation corrections for the energy of each given layer and the LAr-Tile dead material correction from the look-up tables. Apply all corrections according to equations 7.21 and 7.22 .

- Use the iteration for dead material corrections. 


\section{Method validation on Monte Carlo simulation}

Before applying it to beam test data, the calibration is validated on a Monte Carlo sample statistically independent of the one used for extracting the corrections. First, the performance of the compensation weights is evaluated, then the linearity and resolution of the method as a whole. The weighting technique is validated on Monte Carlo simulation samples in separate steps:

- Reconstruct the true deposited energy in the calorimeters (compensation validation).

- Reconstruct the full energy of the incoming particles, including dead material corrections, and quantify the performance in terms of linearity and resolution.

The performance is evaluated in terms of bias and resolution. The weights and dead material corrections are derived from the "correction samples" and applied on the statistically independent "signal samples" (see section 6.3). The results in this section are derived for pions only.

\subsection{Compensation validation}

The reconstructed pion energy after compensation correction is compared to the true deposited energy in the calorimeter. The event-by-event difference $E_{\text {tot }}^{\text {weighted }}-E_{\text {tot }}^{\text {true }}($ calo $)$ is considered, where $E_{\text {tot }}^{\text {true }}$ (calo) is the true total energy deposited in the calorimeter. The bias in the energy reconstruction is defined as the average value $\left\langle E_{\mathrm{tot}}^{\text {weighted }}-E_{\mathrm{tot}}^{\text {true }}(\mathrm{calo})\right\rangle$ and the resolution is obtained by calculating the standard deviation $\sigma\left(E_{\mathrm{tot}}^{\text {weighted }}-E_{\text {tot }}^{\text {true }}(\right.$ calo $\left.)\right)$.

The performance of the LC technique is compared with a simple calibration scheme (called $f_{\text {comp }}$ in the following) which uses beam energy information: each event in the sample is weighted with the same factor $f_{\text {comp }}=\left\langle E_{\mathrm{tot}}^{\text {true }}\right\rangle /\left\langle E_{\mathrm{tot}}^{\mathrm{reco}}\right\rangle$, where $\left\langle E_{\mathrm{tot}}^{\text {true }}\right\rangle\left(\left\langle E_{\mathrm{tot}}^{\mathrm{reco}}\right\rangle\right)$ is the average true total (reconstructed) energy deposited in the given sample in the whole calorimeter, but not in the dead material. The $f_{\text {comp }}$ calibration scheme provides a reference scale to which the improvement in resolution of the $\mathrm{LC}$ weighting can be compared.

The results of the validation procedure are shown in Figure 7. By construction, there is no bias in the energy reconstruction for the calibration procedure using a simple factor. The LC weighting mostly gives a slight positive bias of about $0.6 \%$. At the lower edge of the energy range studied, the bias instead turns slightly negative. The resolution improvement increases with beam energy. It is about $10 \%$ at $50 \mathrm{GeV}$ and about $20 \%$ at $180 \mathrm{GeV}$.

\subsection{Dead material corrections}

Figure 8 shows the bias of the weighted energy, and also the bias of the dead material corrections. For most energies, the LAr-Tile dead material correction has a slight negative bias, while at low energies the bias is positive. The bias is $0.5 \%$ maximally. This cancels out most of the bias from the weighting. The final energy is reconstructed correctly within a few per mil.

\subsection{Linearity and resolution in the Monte Carlo sample}

The performance for the fully corrected energy reconstruction is finally assessed in terms of linearity with respect to the beam energy and relative resolution. The reconstructed energy distribution is fitted with a Gaussian distribution in the interval $(\mu-2 \sigma, \mu+2 \sigma)$, where $\mu$ and $\sigma$ are the mean 


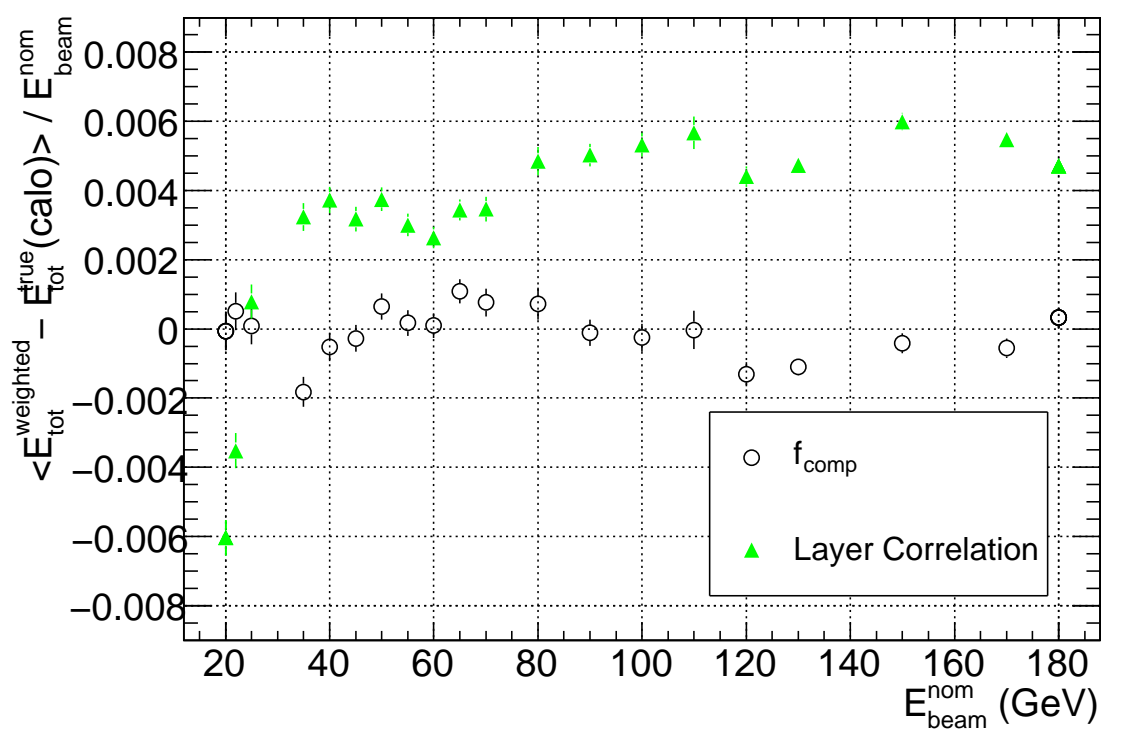

(a)

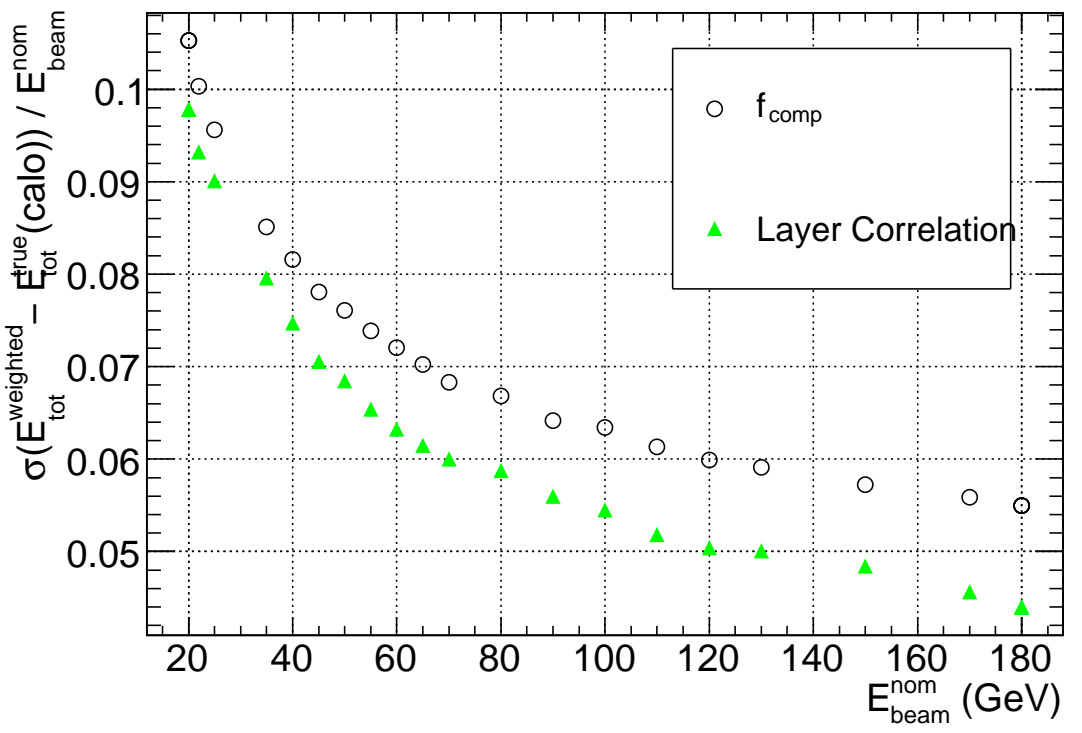

(b)

Figure 7. Bias (a) and resolution (b) of the reconstructed energy after compensation correction minus the true deposited energy for energy deposited in the calorimeters in simulated samples for the calibration procedure using a simple factor and LC weighting.

value and the standard deviation, respectively. This interval is found iteratively. The mean value $E_{\text {fit }}$ and the standard deviation $\sigma_{\text {fit }}$ of the fitted Gaussian are used together with the beam energy $E_{\text {beam }}$ to define the linearity and the relative resolution. 


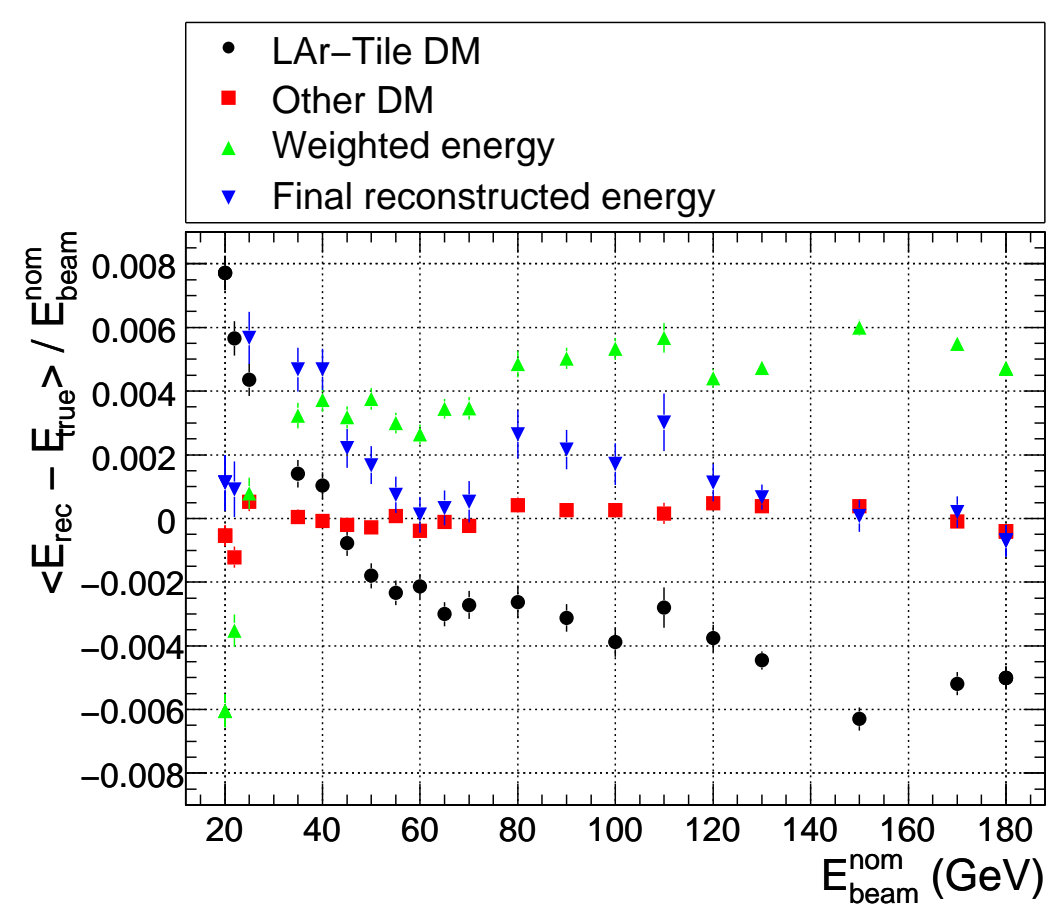

Figure 8. Bias (reconstructed energy minus true deposited energy, divided by beam energy) for the three individual corrections: Weighted calorimeter energy, correction for dead material between the LAr and Tile calorimeters, and other dead material corrections. Lastly, the bias of the final reconstructed energy, which is the sum of the three.

- The linearity is $E_{\mathrm{fit}} / E_{\text {beam }}$ as a function of $E_{\text {beam }}$.

- The relative resolution is $\sigma_{\mathrm{fit}} / E_{\mathrm{fit}}$ as a function of $E_{\mathrm{beam}}$.

Both linearity and relative resolution are derived for the energy distribution at four stages of the energy reconstruction:

- at the electromagnetic scale,

- after applying the compensation weights,

- after compensation weights and application of dead material correction for losses between the LAr and Tile calorimeters, and

- after compensation weighting and all dead material corrections. This last step aims to reconstruct the pion energy.

This is shown in Figure 9. At the electromagnetic scale the calorimeter response is nonlinear-as expected — and only about two thirds of the pion energy is measured. After weighting, between $80 \%$ and $90 \%$ of the incoming pion energy is recovered, while the dead material between the LAr and Tile calorimeters accounts for an additional 8-10\%. After all corrections the correct pion energy is reconstructed within $1 \%$ for all beam energies. Each correction step makes the 
calorimeter response more linear. The compensation weights give a better improvement of the linearity at high energies, while the dead material effects play a more significant role at low energies, in particular at $20 \mathrm{GeV}$ where other corrections than LAr-Tile dead material are important to get to within $1 \%$ of the beam energy. The relative resolution is improved when applying each of the different correction steps. ${ }^{3}$ At high beam energies (above $E_{\text {beam }}=100 \mathrm{GeV}$ ) the contribution of the compensation weights to the improvement in energy resolution has the same magnitude as that of the LAr-Tile dead material corrections. At lower beam energies dead material corrections account for about $70 \%$ of the relative resolution improvement down to about $E_{\text {beam }} \simeq 30 \mathrm{GeV}$. Below $E_{\text {beam }}$ $\simeq 30 \mathrm{GeV}$ all the corrections account for a similar fraction of the improvement: other dead material corrections than those for LAr-Tile account for about $20 \%$ of the resolution improvement, they are marginal above that threshold.

\section{Systematic uncertainties}

Systematic uncertainties on the calibrated energy can be divided into

- The uncertainty of the beam energy: $0.7 \%$ [13].

- The absolute electromagnetic scale uncertainty, which is estimated to be $0.7 \%$ [13] in the LAr calorimeter and 1.0\% [26] in the Tile calorimeter. Scaling the cell energies with their corresponding uncertainties gives a combined electromagnetic scale uncertainty of $0.9 \%$.

- The sensitivity of the results to the proton fraction at each beam energy. It was estimated by varying the fraction used to calculate the corrections. With the assumed fraction adjusted up or down one standard deviation of the TRT measurement, the relative variation in linearity and resolution in data and Monte Carlo simulation was found to be of the order of $1 \%$ for $E_{\text {beam }}=20 \mathrm{GeV}$ and $50 \mathrm{GeV}$ and less than $0.5 \%$ for $100 \mathrm{GeV}$ and $180 \mathrm{GeV}$.

Adding these contributions in quadrature gives a total systematic uncertainty of less than $2 \%$ for each beam energy.

\section{Application of the method to beam test data}

Finally, the method is applied to beam test data, which is compared with Monte Carlo samples with a weighted mixture of pions and protons to match the beam composition.

\subsection{Data to Monte Carlo simulation comparison}

The pion-proton "mixed signal" samples are used to compare data and Monte Carlo simulations in terms of the distribution of the first three components of the layer energy vector along the basis of covariance matrix eigenvectors as defined in section 7.1. Figure 10 shows such a comparison for a proton fraction of $45 \%$ and a beam energy of $50 \mathrm{GeV}$. Good agreement is obtained between data and simulation. The distribution for $E_{\text {eig }, 0}$ shows a double peak structure that separates events mainly showering in the Tile calorimeter from those where the shower starts earlier.

\footnotetext{
${ }^{3}$ The apparent discontinuity in resolution between the results at energies below $150 \mathrm{GeV}$ and those above might be due to a geometry change in the description of the beam test setup: three centimeters of aluminum were included in the Inner Detector system for energies larger than or equal to $150 \mathrm{GeV}$.
} 


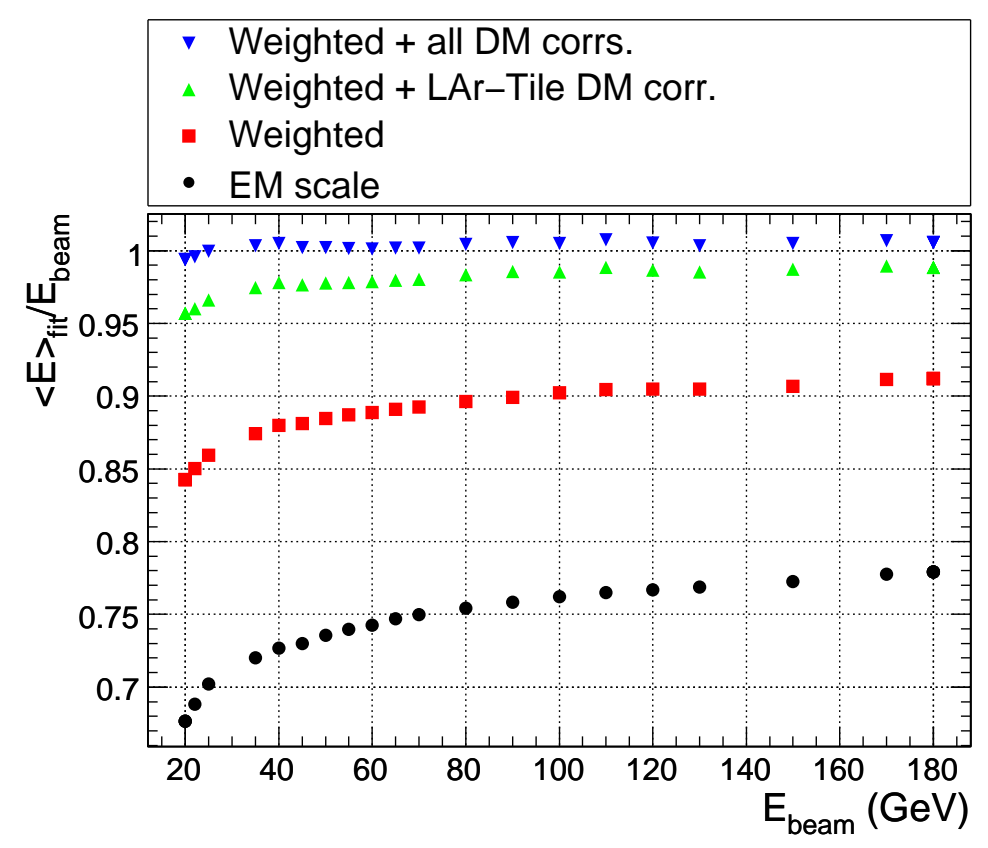

(a)

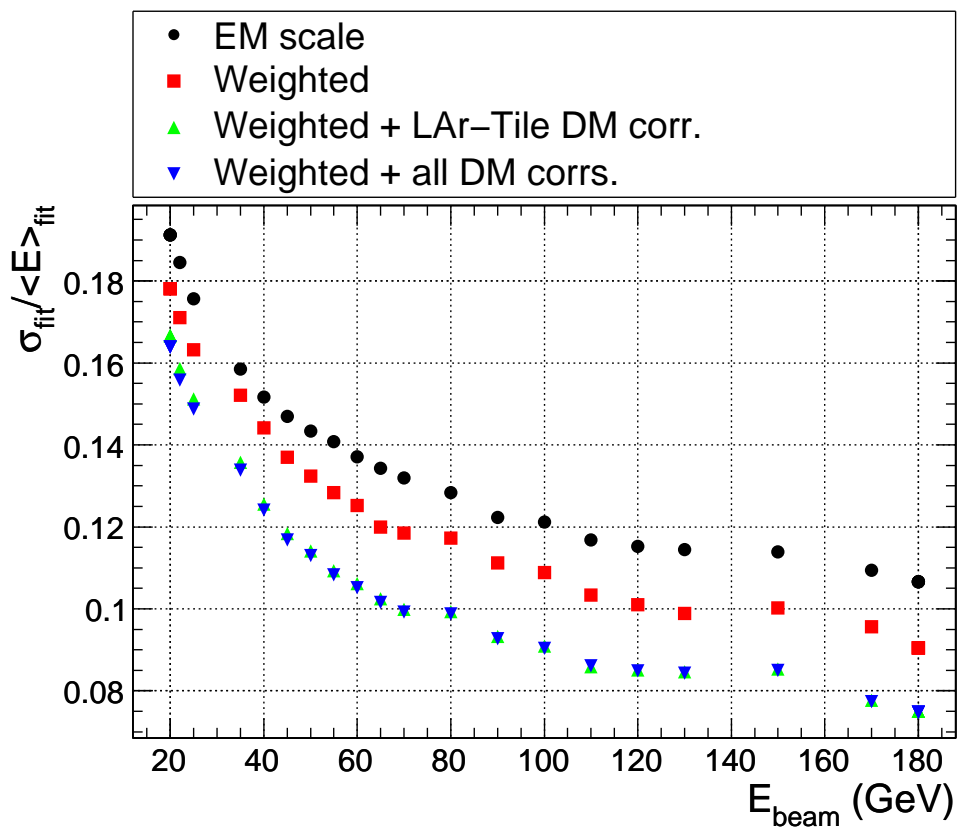

(b)

Figure 9. Linearity (a) and relative resolution (b) for simulated pure pion samples at the electromagnetic scale, with compensation weights applied, compensation weights plus LAr-Tile dead material correction applied, and all corrections applied.

The shapes of the energy distributions (in unit bins of energy and events) for data and Monte Carlo simulation are compared in Figure 11. The corrections are successively applied. Already 


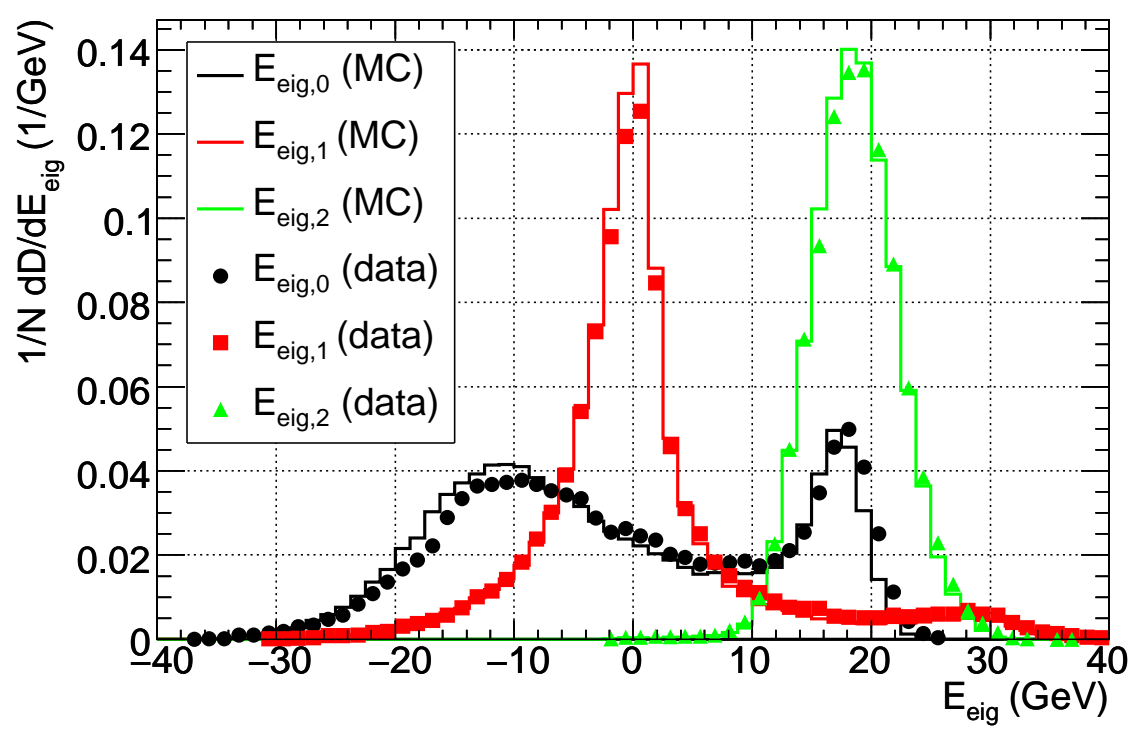

Figure 10. Distribution of the first three eigenvector components for data (filled circles) and Monte Carlo simulation pion-proton "mixed signal" with a proton fraction of $45 \%$ and a beam energy of $50 \mathrm{GeV}$.

at the electromagnetic scale the energy distribution is not well reproduced. The distribution in the Monte Carlo simulation is narrower and less skewed than in the data. This effect is even larger at $20 \mathrm{GeV}$ but less pronounced at higher energies. The quality of the initial description of data by Monte Carlo simulation is not modified by the application of the compensation weights and dead material corrections (see also section 10.2).

\subsection{Linearity and resolution on data}

The performance of the method, as applied to simulation and real beam test data is shown in Figure 12. The data at the electromagnetic scale in this analysis and the one presented in references [11, 12] are in reasonable agreement. The largest deviations of about 3\% (2\%) are seen at $20 \mathrm{GeV}$ $(180 \mathrm{GeV})$. At $20 \mathrm{GeV}$ the difference can be explained by the fact that in that study, the energy in the calorimeters - instead of using topological clustering — was determined by adding the energies $E_{\text {cell }}$ of those calorimeter cells having a pseudo-rapidity within 0.2 of the beam impact point and for which $E_{\text {cell }}$ is two standard deviations above the expected noise. At $180 \mathrm{GeV}$, data in that study were taken with a beam of negatively charged pions, which does not suffer from proton contamination. In addition, for all beam energies data were taken in an earlier run period with a different material configuration upstream of the calorimeters.

The linearity and relative resolution are extracted at all beam energies for both data and "mixed signal" Monte Carlo samples. As in section 8.3 the reconstructed energy distribution is fitted with a Gaussian distribution in the interval $(\mu-2 \sigma, \mu+2 \sigma)$, where $\mu$ and $\sigma$ are the mean value and the standard deviation, respectively. Data (simulation) are shown with markers (horizontal lines) at the electromagnetic scale, with compensation weights applied, with the dead material correction 


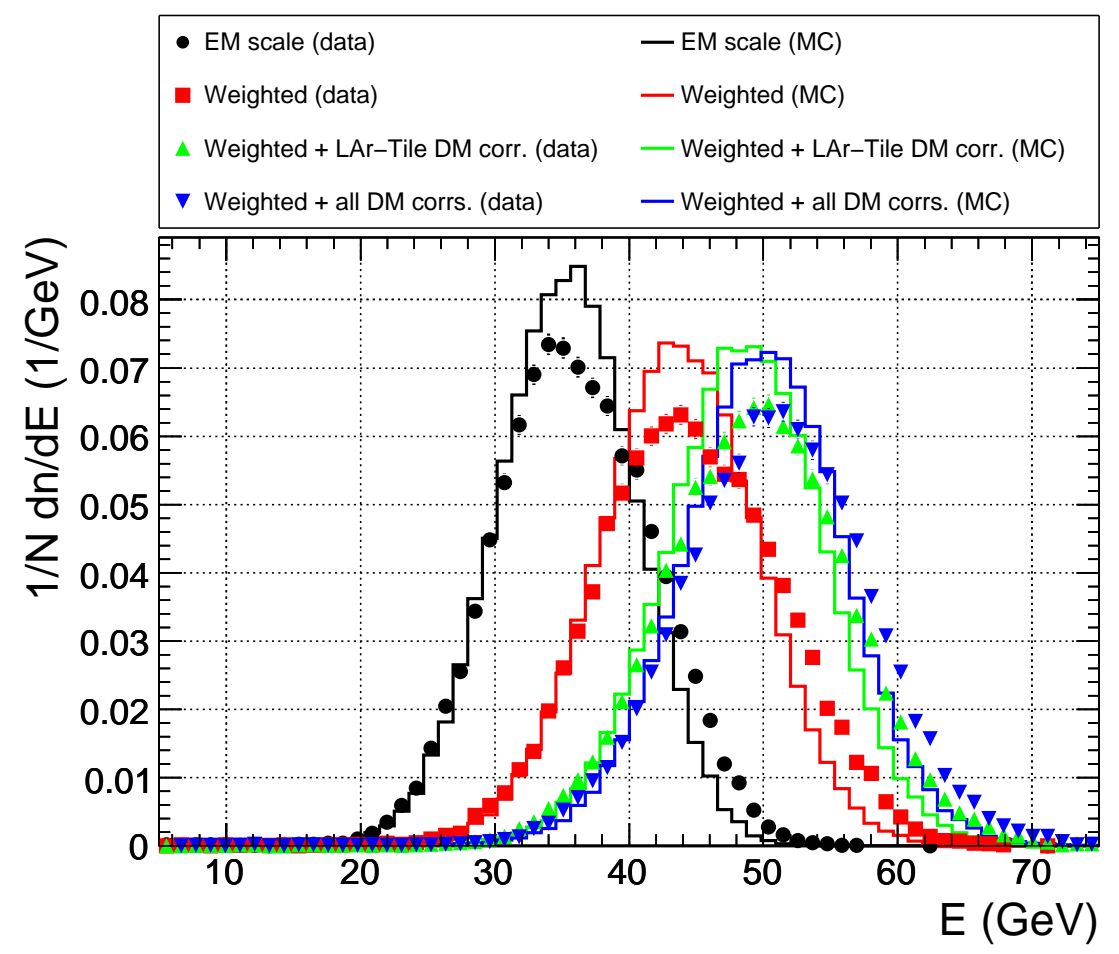

Figure 11. Normalized energy distribution for $E_{\text {beam }}=50 \mathrm{GeV}$ after applying subsequent corrections for compensation and dead material effects. For the Monte Carlo simulation a proton fraction of $45 \%$ is used. .

for energy lost in dead material between the calorimeters applied, and lastly at the final calibrated stage, including all dead material corrections.

After all calibration corrections, the linearity is recovered within $3 \%$ for all beam energies. The discrepancies between data and Monte Carlo are inherited from the reconstructed energy at the electromagnetic scale and they are not considerably changed when the calibration is applied. The relative resolution in data is improved by about $11 \%$ at low energy $(20 \mathrm{GeV})$ and about $25 \%$ at high energy $(180 \mathrm{GeV})$ when moving from the electromagnetic scale to the fully corrected energy scale. A similar relative improvement is obtained in the Monte Carlo simulation: $14 \%$ at low energy and $24 \%$ at high energy. The relative resolution is, however, smaller in Monte Carlo simulation than in data: the discrepancies, at each correction stage, vary between $10 \%$ and $21 \%$ depending on the energy. The relative resolution is smaller in Monte Carlo simulation than in data already at the electromagnetic scale, by about 10-16\%, depending on beam energy. The discrepancies in the shape of the total energy distribution are more pronounced at lower energies and they are already present at the electromagnetic scale.

The ratio of data to Monte Carlo simulation is unchanged within 1\% (4\%) for linearity (resolution) after the corrections are applied. For linearity such changes are of the same order of magnitude as the discrepancies between data and Monte Carlo simulation at the electromagnetic scale: the agreement between data and Monte Carlo simulation is the same for all correction stages. This means that the Monte Carlo simulation is able to predict the corrections that should be applied 


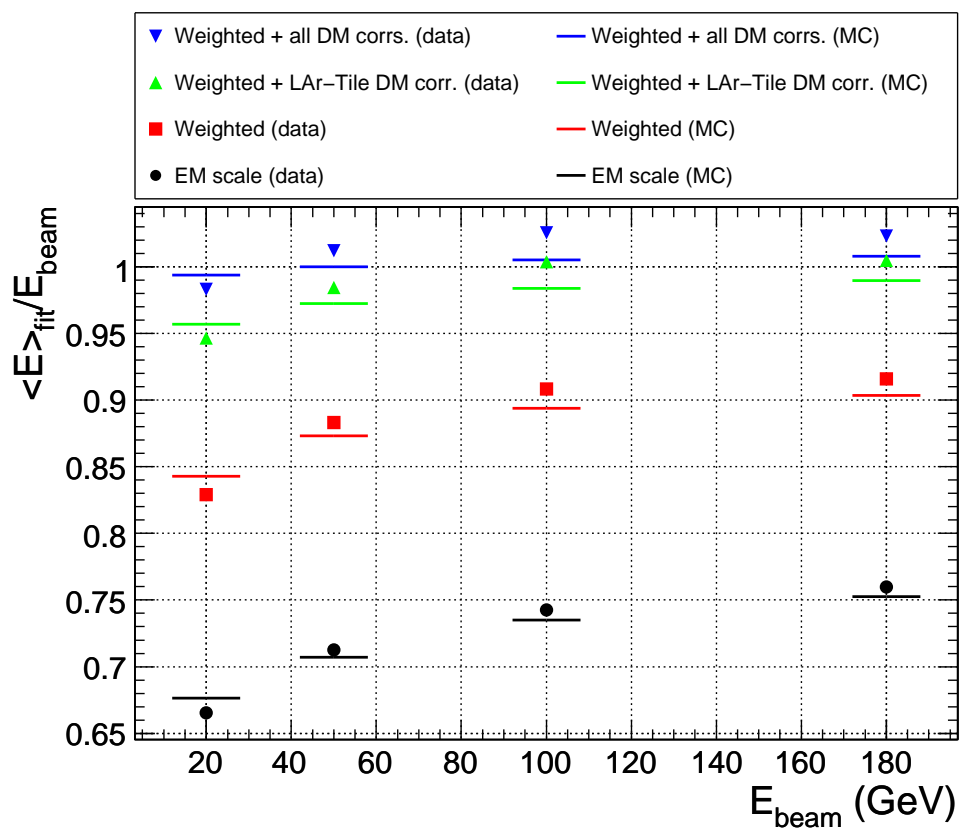

(a)

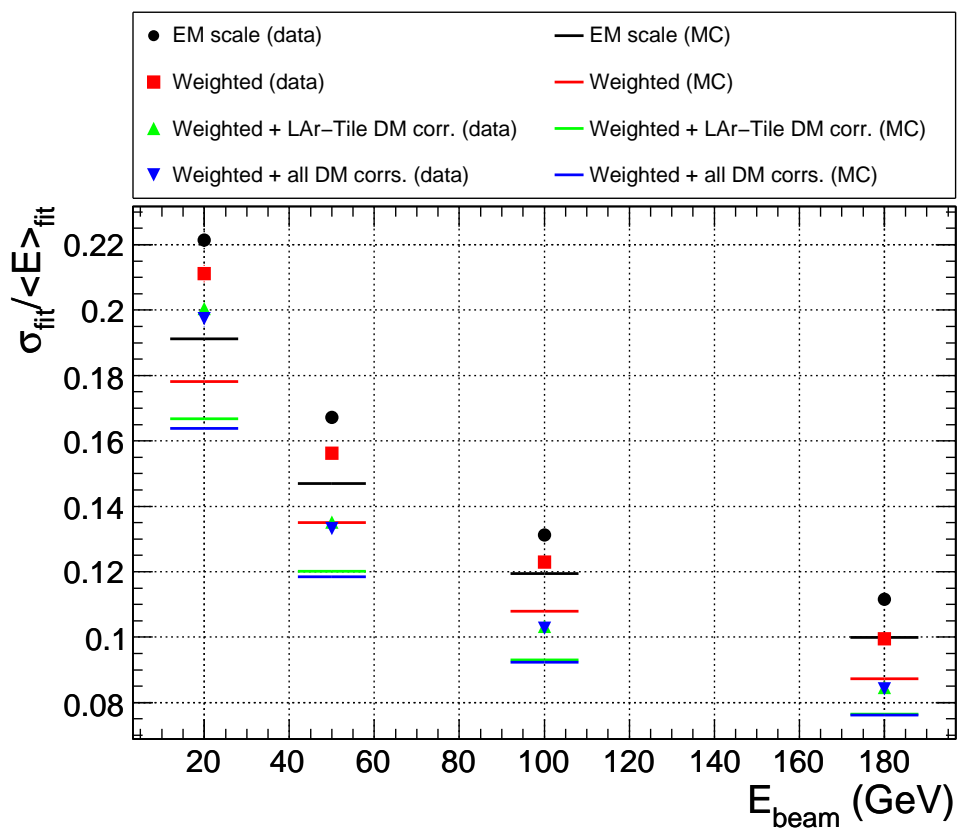

(b)

Figure 12. Data and Monte Carlo simulation are compared for linearity (a) and relative resolution (b) at all stages of the corrections. The markers show data, while the horizontal lines show (a) from bottom to top and (b) from top to bottom: electromagnetic scale, compensation weights applied, weight plus LAr-Tile dead material correction applied, and all corrections applied. See text for details. 
on the data.

The ability of the Monte Carlo simulation to reproduce the data at the electromagnetic scale (i.e., before any correction) seems to be the most critical limiting factor. For the relative resolution the changes are small, if compared with the discrepancies at the electromagnetic scale: the discrepancies do not get worse when the corrections are applied to the data. From preliminary studies a newer Geant4 version (4.9) is able to provide a better, but still not good, description of the resolution in the data.

\section{Conclusions}

An energy calibration technique was developed to deal in a coherent manner with both compensating the hadron response and correcting for the most significant dead material losses in a segmented calorimeter. The technique is based on the sensitivity of the correlation between the deposited energies in the different calorimeter layers to hadronic and electromagnetic deposits.

The calibration technique was successfully applied to the energy reconstruction of pions impinging on a subset of the central ATLAS calorimeters during the ATLAS combined beam test in 2004. When taking into account the beam composition of pions and protons, linearity is recovered within $3 \%$ and relative resolution is improved by between $11 \%$ and $25 \%$. Consistency with the expectation from Monte Carlo simulation studies is good for both the linearity and the percentage improvement in relative resolution. The absolute value of the relative resolution (after all corrections) is larger in data than Monte Carlo simulation by $10 \%$ to $21 \%$.

The discrepancies between data and Monte Carlo simulation are inherited from the reconstructed energy at the electromagnetic scale and they are not considerably altered when applying the calibration. Additional improvement in the data description by Monte Carlo simulation can help to fulfill the expected absolute value for the relative resolution.

\section{Acknowledgments}

A very important ingredient of the 2004 ATLAS Combined Beam Test has been the mechanics of the two calorimeters' support and movement. We would like to thank Danilo Giugni, Simone Coelli, and Giampiero Braga from INFN Milano for the design, overview of the production, and testing of the LAr calorimeter support table. We wish to thank Claude Ferrari, Pierre Gimenez, Yves Bonnet, Denis Gacon, and Alain Pinget of CERN EN/MEF group for the continuous mechanical support provided in the CERN SPS North Area during the installation of the setup and the data taking.

We are grateful to the staff of the SPS for the excellent beam conditions and assistance provided during our tests.

We would like to thank Susan Leech O'Neale for proofreading this paper.

We acknowledge the support of ANPCyT, Argentina; Yerevan Physics Institute, Armenia; ARC and DEST, Australia; Bundesministerium fr̈ Wissenschaft und Forschung, Austria; National Academy of Sciences of Azerbaijan; State Committee on Science \& Technologies of the Republic of Belarus; CNPq and FINEP, Brazil; NSERC, NRC, and CFI, Canada; CERN; NSFC, China; Ministry of Education, Youth and Sports of the Czech Republic, Ministry of Industry and Trade of the 
Czech Republic, and Committee for Collaboration of the Czech Republic with CERN; Danish Natural Science Research Council; European Commission, through the ARTEMIS Research Training Network; IN2P3-CNRS and Dapnia-CEA, France; Georgian Academy of Sciences; BMBF, HGF, DFG and MPG, Germany; Ministry of Education and Religion, through the EPEAEK program PYTHAGORAS II and GSRT, Greece; ISF, MINERVA, GIF, DIP, and Benoziyo Center, Israel; INFN, Italy; MEXT, Japan; CNRST, Morocco; FOM and NWO, Netherlands; The Research Council of Norway; Ministry of Science and Higher Education, Poland; GRICES and FCT, Portugal; Ministry of Education and Research, Romania; Ministry of Education and Science of the Russian Federation, Russian Federal Agency of Science and Innovations, and Russian Federal Agency of Atomic Energy; JINR; Ministry of Science, Serbia; Department of International Science and Technology Cooperation, Ministry of Education of the Slovak Republic; Slovenian Research Agency, Ministry of Higher Education, Science and Technology, Slovenia; Ministerio de Educacion y Ciencia, Spain; The Swedish Research Council, The Knut and Alice Wallenberg Foundation, Sweden; State Secretariat for Education and Science, Swiss National Science Foundation, and Cantons of Bern and Geneva, Switzerland; National Science Council, Taiwan; TAEK, Turkey; The Science and Technology Facilities Council and The Leverhulme Trust, United Kingdom; DOE and NSF, United States of America.

\section{References}

[1] C. Leroy and P. Rancoita, Physics of cascading shower generation and propagation in matter: Principles of high-energy, ultrahigh-energy and compensating calorimetry, Rept. Prog. Phys. 63 (2000) 505.

[2] C. Fabjan and F. Gianotti, Calorimetry for particle physics, Rev. Mod. Phys. 75 (2003) 1243.

[3] R. Wigmans, Calorimetry - Energy Measurement in Particle Physics. Clarendon Press, Oxford, 2000.

[4] D. Groom, Energy flow in a hadronic cascade: Application to hadron calorimetry, Nucl. Instrum. Meth. A 572 (2007) 633.

[5] G. Aad et. al., The ATLAS experiment at the CERN Large Hadron Collider, J. Inst. 3 (2008), no. 08 S08003.

[6] L. Evans et. al., LHC machine, J. Inst. 3 (2008), no. 08 S08001.

[7] Ç. İssever, K. Borras, and D. Wegener, An improved weighting algorithm to achieve software compensation in a fine grained lar calorimeter, Nucl. Instrum. Meth. A 545 (2005), no. 3 803-812.

[8] T. Barillari et. al., "Local hadronic calibration." ATLAS Public Note ATL-LARG-PUB-2009-001, 2009.

[9] N. Nakajima et. al., Correlation matrix method for Pb/Scint sampling calorimeter, in Proceedings of the 2005 International Linear Collider Workshop (J. L. Hewett, ed.), eConf C050318, Mar., 2005.

[10] E. Abat et. al., "Response and shower topology of 2 to $180 \mathrm{GeV}$ pions measured with the ATLAS barrel calorimeter at the CERN test-beam and comparison to Monte Carlo simulations." ATLAS Public Note ATL-CAL-PUB-2010-001, 2010.

[11] E. Khramov et. al., "Study of the response of the hadronic barrel calorimeter in the ATLAS combined test-beam to pions of energies from 20 to $350 \mathrm{GeV}$ for beam impact points from 0.2 to 0.65 ." ATLAS Public Note ATL-TILECAL-PUB-2009-007, 2009. 
[12] E. Abat et. al., Study of energy response and resolution of the ATLAS barrel calorimeter to hadrons of energies from 20 to $350 \mathrm{GeV}$, Nucl. Instrum. Meth. A 621 (2010) $134-150$.

[13] M. Aharrouche et. al., Measurement of the response of the ATLAS liquid argon barrel calorimeter to electrons at the 2004 combined test-beam, Nucl. Instrum. Meth. A 614 (2010) 400-432.

[14] B. Di Girolamo et. al., "Beamline instrumentation in the 2004 combined ATLAS testbeam." ATLAS Public Note ATL-TECH-PUB-2005-001, 2005.

[15] The ATLAS Collaboration, "Response of the ATLAS calorimeters to single isolated hadrons produced in proton-proton collisions at a center of mass energy of $\sqrt{s}=900 \mathrm{GeV}$." ATLAS Conference Note ATLAS-CONF-2010-017, 2010.

[16] The ATLAS Collaboration, "ATLAS calorimeters response to single isolated hadrons and estimation of the calorimeter jet scale uncertainty." ATLAS Conference Note ATLAS-CONF-2010-052, 2010.

[17] The ATLAS Collaboration, "Expected performance of the ATLAS experiment. detector, trigger and physics." CERN-OPEN-2008-020, arXiv:0901.0512v4, 2009. Section 4, p. 309 ff.

[18] J. Jackson, A User's Guide to Principal Components. Wiley, Newark, NJ, USA, 2005. p. 505.

[19] S. Agostinelli et. al., Geant4: A simulation toolkit, Nucl. Instrum. Meth. A 506 (2003), no. 3 250-303.

[20] J. Allison et. al., Geant4 developments and applications, IEEE Trans. Nucl. Sci. 53 (2006) 270-278.

[21] ATLAS Collaboration, "ATLAS Liquid Argon Calorimeter Technical Design Report." CERN/LHCC/96-41, 1996.

[22] ATLAS Collaboration, "ATLAS Tile calorimeter technical design report." CERN/LHCC/96-42, 1996.

[23] K. Nakamura et. al., Review of particle physics, J. Phys. G 37 (2010) 075021.

[24] M. Aleksa et. al., 2004 ATLAS combined testbeam: Computation and validation of the electronic calibration constants for the electromagnetic calorimeter, ATLAS Public Note ATL-LARG-PUB-2006-003, 2006.

[25] W. Cleland and E. Stern, Signal processing considerations for liquid ionization calorimeters in a high rate environment, Nucl. Instrum. Meth. A 338 (1994), no. 2-3 467-497.

[26] P. Adragna et. al., Testbeam studies of production modules of the ATLAS Tile calorimeter, Nucl. Instrum. Meth. A 606 (2009) 362-394.

[27] W. Lampl et. al., "Calorimeter clustering algorithms: Description and performance." ATLAS Public Note ATL-LARG-PUB-2008-002, May, 2008.

[28] P. Adragna et. al., Measurement of pion and proton response and longitudinal shower profiles up to 20 nuclear interaction lengths with the ATLAS Tile calorimeter, Nucl. Instrum. Meth. A 615 (2010) $158-181$.

[29] G. Folger and J. Wellisch, String Parton Models in Geant4, in Proceedings of Computing in High Energy and Nuclear Physics, eConf C0303241, Mar., 2003.

[30] M. P. Guthrie, R. Alsmiller, and H.W.Bertini, Calculation of the capture of negative pions in light elements and comparison with experiments pertaining to cancer radiotherapy, Nucl. Instrum. Meth. 66 (1968) 29-36.

[31] H. W. Bertini and M. P. Guthrie, Results from medium-energy intranuclear-cascade calculation, Nucl. Phys. A 169 (1971) 670-672.

[32] N. Stepanov, "Statistical simulation of high-excited nuclei fission. II. calculation and comparison with experiment." Preprint, 1988. ITEP-55 (Moscow). 
[33] "Geant4.7 physics reference manual."

http://geant4. cern. ch/support/userdocuments. shtml, 2005. 